\title{
I porti del grano siciliano nel XVIII secolo
}

\author{
Antonino Blando
}

\section{PORTI, LA GUERRA E IL GRANO}

La mattina del 26 dicembre 1798, dopo una burrascosa fuga e un tempestoso viaggio, nel porto di Palermo sbarcava, su un vascello di guerra inglese comandato dall'ammiraglio Nelson, la famiglia reale borbonica in fuga da Napoli. La penisola ormai era in mano alle truppe francesi del generale Championnet e la Sicilia così diventava uno strategico teatro di guerra nel Mediterraneo. Il principe De Luzzi già nel maggio di quel fatidico 1798, aveva incominciato ad organizzare, nella sua altissima carica di viceré di Sicilia, la «milizia urbana e la gente armata dei baroni" per difendere l'isola - ormai posta sotto il protettorato militare inglese - dall'attacco delle truppe francesi. La situazione era drammatica : venivano requisiti e confiscati tutti i bastimenti e le proprietà dei mercati francesi o genovesi, si ordinava la costruzione di nuove navi da guerra, si acquistavano fucili e cannoni, si impiantavano fabbriche belliche, si cercava di preparare ed equipaggiare alla meno peggio un esercito raccogliticcio e, infine, si fortificavano i porti e le coste ${ }^{1}$.

Il nemico poteva arrivare da un momento all'altro. La Calabria era distante un braccio di mare e, come se non bastasse, i francesi occupavano l'isola di Malta. La difesa dei porti diventava la priorità del governo. Al marescialli Persichelli veniva affidato il compito di fortificarne le difese. Il 6 febbraio inviava gli ordini per «provvedere efficacemente alla valida difesa di questo Regno della Sicilia, onde renderlo immune da qualunque

1. Cfr. A. De Francesco, La Sicilia negli anni rivoluzionari e napoleonici, in E. Iachello (a cura di), I Borboni in Sicilia (17341860), Catania, 1998.

2. A. Sansone, Gli avvenimenti del 1799 nelle due Sicilie, Palermo, attacco ostile». Si ordinava il riarmo delle piazze di Messina, Palermo, Milazzo, Patti, Trapani e Palermo; ma prima di tutto veniva dato ordine di «impedire uno sbarco nei Caricatori di Termini, Girgenti, Licata, e Sciacca. Dove si formi una batteria provvisionale di cannoni per ciascuno nei siti convenienti in modo che tali caricatori siano guardati dagli attacchi marittimi dell'inimico $»^{2}$.

La grande politica europea, la rivoluzione, il teatro di guerra, i ruolo geo-politico della Sicilia : cosa univa i caricatori a tutto questo, tanto da doverli difendere con "batterie di cannoni»? La risposta era antica e nuova allo stesso tempo. I caricatori custodivano da secoli il grano, la risorsa, materiale e simbolica, più importanti dell'isola. In Sicilia la politica, così come la guerra, ha sempre parlato il linguaggio del grano ${ }^{3}$.

\section{IL MAESTRO PORTULANO}

Il Maestro Portulano, istituzione di origine aragonese, si occupa per oltre mezzo millennio, sino al 1823, di gestire i permessi di esportazione del grano (tratte) concessi dal governo e di controllare il sistema statale dei porti specializzati in questo commercio (caricatori). Nell'evoluzione storica del mercato del grano, questa l'istituzione gioca un ruolo cruciale nel condizionare il comportamento e l'agire economico dei soggetti in esso coinvolti. Il mercato di antico regime è sempre un sistema vincolato di commercializzazione e più che mai lo è uno come quello del grano legato alla stessa legittimazione politica del

1901 (Documenti per servire alla Storia Patria di Sicilia), p. 29.

3. Anche e soprattutto nei momenti di grande tensione politica, si veda S. Laudani, "Quegli strani avvenimenti». La rivolta di Palermo del 1773, Roma, 2005. 
governo. Quest'ultimo deve garantire il fabbisogno dei sudditi (la triade "Pane, Popolo e Re» è, infatti, alla base di contratto sociale paternalistico di antico regime ${ }^{4}$ ), ma nello stesso tempo deve poter assicurare ai mercanti la maggiore commercializzazione possibile del grano, perché da ciò proviene uno dei cespiti fondamentali delle finanze statali, e ancora deve tener presente le richieste dell'aristocrazia latifondista produttrice di grano ${ }^{5}$.

I caratteri originari che il mercato assume nell'epoca tardo medievale si conservano lungo l'intera vicenda del grano siciliano : l'intervento dello stato che utilizza il grano come grande risorsa politico-fiscale, la priorità del circuito interno del grano rispetto a quello estero, la domanda internazionale che stimola l'aumento dei prezzi e la specializzazione dell'agricoltura, un mercato interno integrato su basi anti-vincolistiche, lo sviluppo di sistemi di credito che fissano i prezzi alla produzione (mete), il ruolo arbitrale svolto dal governo nella determinazione dei diritti doganali, l'istituzionalizzazione del mercato del grano tramite la creazione di un'agenzia specializzata (il Maestro Portulano) che gestisce centralmente un efficace sistema portuale di stoccaggio del grano (i caricatori) disseminato lungo le coste della Sicilia a breve distanza dai luoghi di produzione e, se vogliano, anche vicino ai luoghi di consumo internazionali.

Il Maestro Portulano, che è quasi sempre un grande mercante, controlla anche un'altra risorsa fondamentale: l'informazione. Risorsa preziosa tanto al governo quanto ai mercanti. La centralizzazione degli affari a Palermo, da dove si tratta il commercio estero del grano dell'intera isola, permette la creazione di un mercato speculativo (determinato dai prezzi internazionali) delle tratte e dei certificati di deposito del grano nei caricatori. Deposito garantito dal Maestro Portulano e quindi dallo stato.

Il grano è una merce preziosa, pesante, ingombrate e facilmente deperibile. Nell'isola, diversamente che in Italia settentrionale e nel nord Europa, si tendeva a coltivare, più che il grano tenero (maiorca), il grano duro (roccella). Capace di conservarsi a lungo inalterato e quindi meglio adatto al commercio. Per venire immerso nel mercato, il grano aveva bisogno di essere traspor-

4. Si rimanda ai classici di S. Kaplan, Bread, Politics and Political Economy in the Reign of Louis XV, The Hague, 1976. Id., Le complot de famine : histoire d'une rumeur au XVIII' siècle, Parigi, 1982. tato sin ai porti. Operazione costosa ma relativamente semplice nei mesi successivi il raccolto, quando le strade sterrate (chiamate «trazzere») non erano spazzate via dalla pioggia invernale $\mathrm{e}$ potevano essere percorse dalle carovane di muli carichi di sacchi di grano. Era quindi preferibile da parte dei produttori ammassare il grano nei magazzini dei porti, in modo da consentire, in qualsiasi parte dell'anno, uno scambio più efficiente con i committenti. Il porto era quindi l'anello più robusto che tiene assieme da un lato i produttori e dall'altro i mercanti.

\section{CHE COS'È UN CARICATORE?}

La gestione di questi magazzini, chiamati «caricatori» (perché da lì il grano veniva poi caricato sulle navi) era affidata al Maestro Portulano. Il grano veniva separato dalle altre merci e sottoposto ad una tutela particolare: da una parte c'era il porto dall'altra il caricatore. Qui il grano veniva custodito $o$ in fosse $o$ in grandi magazzini dove non solo si conservava bene ma, sfruttando il suo naturale aumento di peso (la crescimonia) e giocando tra il peso iniziale e quello finale, il Portulano poteva piazzare sul mercato quantità ingenti di derrate. Dato che il deposito non si pagava, erano le crescimonie l'unico provento che il caricatore riceve per la manutenzione del grano; poiché la crescita di peso si verifica solo il primo anno, se esso non veniva venduto entro tale termine i proprietari erano soggetti al pagamento di un tarì a salma $(=220 \mathrm{Kg})$ per la custodia, per un altro anno. I caricatori regi inoltre godevano del "mero e misto imperio", cioè di una giurisdizione privilegiata che li rendeva autonomi dalle leggi e dai tribunali delle città. I caricatori regi dipendevano direttamente dal Maestro Portulano, ed erano quelli storicamente più importanti dell'isola; gli altri dipendevano dalle istituzioni delle città demaniali o da quelle feudali. In tutte e due i casi non si poteva esportare (estrarre) grano senza il permesso del Maestro Portulano. Le procedure da eseguire erano standardizzate per tutti i caricatori e prevedevano una certificazione del deposito e la garanzia della qualità del grano conservato.

A capo di ogni caricatore vi era un viceportulano affiancato dal "portulanotto", da un

5. Cfr. I. Fazio La politica del grano. Annona e controllo del territorio in Sicilia nel Settecento, Milano, 1993. 
«maestro notaro» e poi da una serie di figure intermedie che andavano dai custodi dei magazzini, ai "paliatori» del grano, ai «travaglianti» che scaricavano il grano in arrivo dall'entroterra sulle some dei muli per poi caricarlo sulle navi in partenza. Così la "corte" dell'ufficio del Maestro Portulano si componeva, alla fine del XVIII secolo, di 760 unità impiegate nei quattro caricatori regi (Sciacca, Girgenti, Licata, e Termini a i quali se ne affiancheranno a fine Settecento altri due - Terranova e Catania - di minore importanza) e negli altri ventidue feudali o demaniali (Palermo, Castellammare del Golfo, Trapani, Marsala, Mazara, Vindicari, Agnone, Agosta, Siracusa, Acireale, Aci S. Antonio e Filippo, Taormina, S. Alessio, Gallidoro, Milazzo, Patti, S. Marco, Brolo, Tusa, Caronia, Roccella, Bonfornello, Cefalù, Lipari e Ustica) ${ }^{6}$.

A centro, cioè a Palermo, come nel resto dell'isola, le cariche di Maestro Portulano, che diventava vice nei diversi caricatori, e del Maestro notaio, che certificava le decisioni, erano vendibili. Tutti gli altri impiegati lo erano dopo la "provvisione» del Maestro Portulano, ad eccezione di Palermo e Castellammare. Nella capitale il Maestro notaio, il revisore, il capo dei misuratori, i due guardiani e i ventidue misuratori erano nominati dal Senato della città; mentre a Castellammare gli altri quarantadue dipendenti del caricatore li nominava il Principe di Aragona. Il numero più alto di impiegati era concentrato nei quattro grandi caricatori da dove partivano la maggior parte delle esportazioni di grano per «infra e

6. Vedi la mappa dei caricatori nel saggio di S. Laudani in questo volume.

7. Archivio di stato di Palermo (d'ora in poi ASPA), Real segreteria incartamenti, b. 5253 cc. nn. E ancora : "alzatori di sacchi, aggiustanti seu bastasi per il trasporto del frumento dai magazzini al ponte; barcaroli per il trasporto con barchette del frumento o altro dal ponte ai vascelli; coffiatori per lo scarcico delle coffe piene di frumento dalle barche ai vascelli; pontiggieri per la sorveglianza al ponte per evitare furti o danni; tendigieri per montarela tenda che casca sopra le barche per non buttarsi frumento a mare; rimorchio per guidare la barchetta che rimorchia le altre barche dal ponte al vascello; cavallitto per montare un cavallitto con chianche e tavole quando il ponte è carico; sollevatori per istradare li padroni che vengono a caricare ed avvisare l'officiali e mercanti che hanno intervenire al carico; misuratori; detentori di libri; guardiani di spiaggia, mastro garzoniere; tumminiero; conservatore di sacchi». In, Privilegi e prerogative del Maestro Portulano (1714), in Biblioteca Comunale di Palermo, extra" regno : solo Girgenti ne contava ben novanta: "Vice portulano, Maestro notaio, tre portulanotti estrattori, governadore magazziniere, consultore, fiscale, primo detentore, secondo detentore, primo e secondo aiutante del detentore, giovane aiutante, revisore di frumenti, ministro, mandatore, tre portulanotti ricevitori, sei ricevitori, pontigiero, piombero, serviente della detentoria, capo dei misuratori, tre portieri, trenta misuratori, capo di paliatori, diciotto paliatori, garzonotti di corte, tumminiero, chiavittiero e cinque guadiani di porto e marine ${ }^{7}$.

I caricatori funzionano, quindi, come gli sportelli periferici di un istituto centrale con lo scopo di raccogliere le risorse e ridistribuirle secondo precise direttive che vengono dal vertice. Un sistema che ricorda le forme dell'economia di «ridistribuzione» centralizzata di cui parla Karl Polanyi $^{8}$. È significativo che nel dibattito sulla libertà di esportazione del grano della seconda meta del Settecento non compaiano mai i caricatori che, in fondo, del vincolismo sono l'espressione più manifesta. Ciò perché non solo lo stato, ma anche i produttori e i mercanti avevano tutto l'interesse che questo sistema funzioni; tanto che, nelle annate buone, quando si riempiono i magazzini, sono gli stessi baroni a chiedere e a farsi carico delle spese per scavare nuove fosse nel caricatore dove depositare il grano9. I mercanti trovavano già pronto il grano all'imbarco e non dovevano andare a prelevarlo nei feudi dell'interno, e inoltre avevano la possibilità di contrattare centralmente i loro affari garantiti
Ms Qq F 110, cc. nn.

8. Cfr. K. Polanyi (a cura di), Traffici e mercati negli antichi imperi, Torino, 1978 (ed. orig. 1957), p. 297-331.

9. A Termini si prendevano in affitto diversi magazzini adiacenti a caricatore quando le 13 mila salme della sua capacità normale non riuscivano a contenere tutto il grano che scendeva dalle montagne. Don Saverio Palmeri scriveva, nel 1789, al Maestro Portulano che era disposto a farsi carico di tutte le spese per la custodia del grano in magazzini privati che rispondessero a tutte le "costumanze" di qualità richieste, pur di poter esportare il grano. ASPA, Maestro Portuiano (d'ora in poi MP) b. 1188 cc. nn. Così come il marchese Giuseppe del Bosco Branciforti, l'anno precedente, aveva chiesto la possibilità "di approntare a sue spese" un magazzino, dove poter immettere 800 salme di frumenti già venduti, provenienti dai suoi Stati di Castrofilippo e Realmonte, che non trovavano spazio nel caricatore di Girgenti. ASPA, MP, b. 1187 cc. nn. 
dall'istituzione del Maestro Portulano. Lo stato aveva la possibilità in ogni momento di chiudere l'esportazione dai caricatori in presenza di annate scarse; inoltre disponeva di una riserva di grano pronta per ogni emergenza.

\section{COME FUNZIONA UN CARICATORE}

L'ordinamento di questi veri dockes e banchi frumentari nello stesso tempo, notava l'economista Giuseppe Salvioli all'inizio del'900, era complicato e minuzioso, come tutte le cose del governo vicerale. [....] Tenevasi diligente annotazione del grano che entrava e di quello che si estraeva per l'interno e per l'estero. Ai proprietari si rilasciavano certificati o cedole di deposito seguito, delle responsali perfettamente identiche ai moderni warrants, che si potevano vendere e pignorare, e sulle quali si faceva operazioni di credito alle tavole e nelle banche private ${ }^{10}$.

Compito fondamentale dell'amministrazione, secondo le «istruzioni», era di garantire i mercanti.

Perchè della buona qualità de formenti nasce l'introduzione del commercio in questo Regno e dallo stesso ne resulta non solo l'utile per la Regia Corte m'anche il commodo e sollievo del Borghesato, e del Commune Pubblico, abbiamo stimato per aumento del medesimo stabilire e piantare una buona regola per l'amministrazione del Caricatore di Trapani.

Così iniziavano "le istruzioni" che il Maestro Portulano, Antonio Lucchese Duca della Grazia, emanava il 22 novembre 1726 "per il buon governo del nuovo caricatore di Trapani $»{ }^{11}$. Istruzioni che da secoli seguivano, più o meno fedelmente, tutti gli altri caricatori.

Che l'officiali a chi spetta far la recezione de formenti debbano quelli ricevere di buona qualità, netti, asciutti e ben crivellati mercantabili e recettibili senza tollerare alcun vizio umano nell'accettazione delli stessi $[\ldots]$ ed usandosi la nessuna ammissione o dissimulazione che si vogliono [...] da tal buona recezione dipende il credito del Caricatore a considerazione del quale s'animeranno maggiormente li negozianti forestieri a stabilire il lor traffico.
E per garantire la fiducia dei mercanti era necessario garantire la qualità del grano conservato nei magazzini, che doveva essere sottoposto a un minuzioso e scrupoloso controllo a campione. Se non si superava questa verifica il grano doveva essere rifiutato.

S'accaderà, che nelli formenti, che habassero nel caricatore per riceverli si trovassero cocci punto nella crivata di mezzo tomolo che farsi prendere a dal Garzone di Corte, in tal caso se in detta crivata il suo punto ascendersi a cocci due o pur tre e non più sia lecito allora all'officiali di poterli ricevere e trovandosi cocci quattro o più sino a dieci sia lecito di farsene cernere e scapuzare salma una e se osserverà che il punto sudetto si leverà col crivo, sia lecito di farsi cernere tutto dell'istessa maniera e farsene la recezione e non sevandosi che si debba ossolutamente rimandare. Se però il sudetto punto rileverà più delli cocci dieci si dovrà li detti formenti rifiutare adirittura e procede tutte le volte il formento che habbasterà avrà tutte l'altre buone qualità e mancandone una tanto, il primo punto, anche le meno fosse quanto per il vizio che avrà sempre si dovrà rimandare, o rifiutare.

Il frumento doveva essere non solo pulito da pietre e terra, ma anche da altri semi che ne potevano compromettere la qualità e la conservazione.

S'accaderà che pigliandosi la crivata dal Garzone di Corte nel formento che abbasterà per riceversi è nello stesso se il troveranno escrementi di sotto di gioglio, terra o altre semenze che si potessero fare vizio o pur di sopra, come sarebbe l'orzo, il fasolo salvatico, ed altro, che pura potrebbe farci vizio, in tal caso trovandosi di escrementi di sotto la loro piena, che appresso l'officiale esiste, allora li formenti dovranno cernersi per farsene la recezione, per l'escrementi che potranno trovarsi di sopra dovransi pure cernere è scapuzare per farsene parimenti la recezione avendo tutto ciò luongo tutte le volte che li formenti sudetti si troveranno asciutti senz'alcun vizio umano è non trovandosi tali, si dovran rifiutare e rimandare e per ingordi e per lordi $[\ldots]$

Che dal principio del ricevimento dei formenti di qualsivoglia raccolto per tutto il mese d'agosto d'ogni anno non possono l'uff.li Ricevitori ricever 
formenti che non fossero netti, asciutti e ben crivellati, mercantibili e recettibili, ed essendo inqualche parte ingordi, che si debbano assolutamente rifiutare, proibendoli alli sud. Ufficiali di riceverli, etiam che dal Bordonaro, o Padrone del formenti li fosse offerto l'ingordo per non darsi luogo alle frodi.

Esercitando il "mero e misto impero», il viceportulano poteva ordinare l'arresto del bordonaro (cioè del trasportatore del grano) quando tentava di depositare un frumento di cattiva qualità. Inoltre si doveva prendere nota del nome del produttore del frumento e da dove arrivava per controllare meglio le partite future.

Venendo qualche imposta di formento per ricevere [...] vizio, come s'è detto di sopra che debba subito lo Bordonaro ritornarselo e riportarselo e non facendolo al più per lo spazio d'ove sei che si voglia dall'officiali far mettere carcerato à nome del Viceportulano a cui poscia se ne dovrà partecipare la notizia per gastigarlo a vista della milizia, che sarà privedentemente interpetrata, dovendo l'off.li ricevitori notare nella fine de loro libretti la rifiutata del sudetto formento rimandato con descrizione la giornata, il nome del Padrone, la quantità, il nome del Bordonaro, è la dove lo porterà, per starsi con più oculatezza venendo altro formentto dell'istesso Padrone è del medesimo luogo.

Minuziosa doveva essere pure la compilazione e conservazione dei registri.

Che ogn'uno dell'off.li ricevitori dovrà tenere il suo quinterno separato è nel medesimo dovrà notare il giorno è la quantità del formento che si riceverà prima in lettera stesa, il nome del padrone quello del Bordonaro, il luogo d'onde viene ed il magazzino o fossa ove si immetterrà, proibendosi all sudetto officiali potersi copiare partita l'un dall'altro libro $[\ldots]$ Che sia proibita all'off.li uscir Bollettino dupplicato p. una partita di formento ricevuto nel caso che dal Bordonaro o altro li fosse ricercato con dire d'averlo deperso o smarrito per altro accidente ne meno con direvaglia per una volta tantum, ma in tal caso dovran far riccorso dal detentore per uscirgli la fede di tal partita ricevuta, come stà nel Giornale, dovendo prima precedere fideiussore per l'atti dell'officio di Maestro Notaro di non doversi sudetto Bordonaro, o altro, letare del bollettino forse ritrovato, stante la fede uscita di tal partita.
Una scrittura esatta dei registri era indispensabile perchè il grano doveva essere restituito ai compratori nella quantità e qualità con cui era stato depositato.

Che debbiano l'officiali specificare ne loro quinterni la qualità de form.ti se siano forti o roccelli, e così ne debbiano uscire li bollettini a fine d'ogni Padrone potessi sorvire del suo e doverseli restituirli come li han mandati, cioè forti per forti e roccelli per roccelli [...] Che debbino li uff.li ricevere separatamente li formenti forti dalli roccelli e debbiano similm.te metterli in magazzini diversi cioè in un magazzino li forti e in un altro li roccelli $[\ldots]$ Che non possino nel caricare li bordonari mescolar formenti d'un padrone all'altro e farne una sola recezione [...] Che empita sarà una fossa si vogli prima dall'ufficiali comprobare la quantità de li form.ti, e tirando d'accordo voglino fare una poliza nella quale dovranno descrivere il nome dell'istessa, la quantità appostasi, i nomi de misuratori che la mesurarno, confirmandola con loro proprio pugno e doppo in presenza del Viceportulano metterla dentro un cannolo che dovrà collacarsi in detto formento e poscia suggellarsi la fossa sudetta con ammonte collarvisi terra, per poi quando dovrà di sfossarsi, osservarsi se si troverà corrispondente colla quantità già immessa.

La manutenzione dei magazzini doveva essere costante per verificare lo stato dei frumenti e poterlo mescolare con le pale. Il controllo era affidato a un "Governatore Magazziniero" che doveva gestire pure un complicato sistema di uso delle chiavi dei magazzini. Inoltre il Governatore

non debba ricever polize o atti mandatarj fatti per gli atti di notari forestieri se prima non sarà la firma del mandante o l'estratto del nostro da due persone degne di fede riconosciute $[\ldots]$ e si proibisce di ricever polize firmate da donne, anche che fossero avverate e riconosciute.

Era proibito agli impiegati «direttamente o indirettamente ingerirsi in negozj formentarj, mantenimento di redine per condurre frumenti o altri negozio simile».

Tutti i movimenti del caricatore venivano riportati su tre registri separati. Uno del viceportulano, uno del notaio e infine uno per il potulanotto che ogni quattro mesi dovevano essere spediti a Palermo «separatamente», proibendo "ad'ogni uno degli 
officiali suddetti di copiarsi una partita d'un altro, per non darsi il caso ch'errando errassero tutti e l'istesso dovranno osservare tutti colli scaricati, facendo l'istessa rubrica colle sue negative inpiedi" .

Le estrazioni di grano per Palermo e Messina avevano una formalità a cui deve adempiere personalmente il viceportulano :

consignare al Padrone del bastimento una quantità di frumento in una cassetta ben suggellata in presenza dello stesso, per servirci di mostra e costare sempre della buona qualità e non darsi luogo alle frodi ed alterazioni che potrebbero commettere, dovendo anche il sudetto Viceportuno trattenersene la mostra nell'officio per costar sempre la qualità del primo formento già caricato.

Cura fondamentale del Viceportulano doveva essere infine quella di «repurtare e trattare con inspeciale favore li padroni de li bastimenti che verranno nel caricatore per maggiormente animarsi allo mercio, ed introdurre negozi per l'accerto della Regia Corte, commodo del Borghesato, ed utile del pubblico».

\section{IL SETTECENTO SECOLO DEL GRANO PER «EXTRA REGNO»}

Il grano che tra il 1720 il 1823 parte dai caricatori della Sicilia ammonta complessivamente a 14.329.662 salme di cui il $70 \%$ (pari a salme 9.892.288) per l'estero (vedi tav. 1), e il resto diretto "infra Regno", cioè verso i porti delle altre città dell'isola, per un totale di 4.437.364 salme $^{12}$.

La crisi dei primi decenni del XVIII secolo viene subito superata e già nel 1722-23 si « estrag-

Esportazione di grano per «extra Regno» (1720-1832)

(Valori in salme $=222 \mathrm{~kg}$ )

\begin{tabular}{|c|r||c|r|}
\hline ANNI & EsPORTAZIONI & ANNI & EsPORTAZIONI \\
\hline $1719-20$ & 5.768 & $1767-68$ & 25.157 \\
$1720-21$ & 31.742 & $1768-69$ & 184.217 \\
$1721-22$ & 61.619 & $1769-70$ & 173.873 \\
$1722-23$ & 125.096 & $1770-71$ & 271.178 \\
$1723-24$ & 128.828 & $1771-72$ & 43.701 \\
$1724-25$ & 94.061 & $1772-73$ & 122.640 \\
$1725-26$ & 93.077 & $1773-74$ & 69.089 \\
$1726-27$ & 93.967 & $1774-75$ & 16.992 \\
$1727-28$ & 108.726 & $1775-76$ & 156.011 \\
$1733-34$ & 142.067 & $1776-77$ & 204.350 \\
$1734-35$ & 185.986 & $1777-78$ & 137.047 \\
$1735-36$ & 101.135 & $1779-80$ & 149.093 \\
$1736-37$ & 41.018 & $1780-81$ & 150.899 \\
$1737-38$ & 188.846 & $1781-82$ & 293.460 \\
$1738-39$ & 179.771 & $1782-83$ & 72.157 \\
$1739-40$ & 191.404 & $1783-84$ & 75.225 \\
$1740-41$ & 145.341 & $1784-85$ & 67.347 \\
$1741-42$ & 125.435 & $1785-86$ & 269.562 \\
$1742-43$ & 143.733 & $1786-87$ & 98.911 \\
$1743-44$ & 73.924 & $1787-88$ & 141.714 \\
$1745-46$ & 111.666 & $1788-89$ & 265.412 \\
& & &
\end{tabular}

(segue)

\begin{tabular}{|c|r||c|r|}
\hline \multicolumn{1}{|c|}{ ANNI } & EsPORTAZIONI & ANNI & EsPORTAZIONI \\
\hline $1746-47$ & 103.684 & $1789-90$ & 295.948 \\
$1747-48$ & 26.796 & $1790-91$ & 40.021 \\
$1748-49$ & 70.367 & $1791-92$ & 78.906 \\
$1749-50$ & 206.585 & $1792-93$ & 3.100 \\
$1751-52$ & 302.984 & $1793-94$ & 405.769 \\
$1752-53$ & 153.699 & $1794-95$ & 258.944 \\
$1753-54$ & 128.961 & $1795-96$ & 291.059 \\
$1755-56$ & 54.915 & $1796-97$ & 162.435 \\
$1756-57$ & 109.873 & $1798-99$ & 39.344 \\
$1757-58$ & 223.288 & $1803-04$ & 35.427 \\
$1758-59$ & 144.337 & $1805-06$ & 910 \\
$1759-60$ & 166.746 & $1806-07$ & 44.489 \\
$1760-61$ & 11.194 & $1808-09$ & 12.112 \\
$1761-62$ & 55.643 & $1813-14$ & 12.965 \\
$1762-63$ & 58.224 & $1814-15$ & 7.531 \\
$1763-64$ & 43.299 & $1817-18$ & 48.143 \\
$1764-65$ & 365.755 & $1818-19$ & 71.392 \\
$1765-66$ & 440.267 & 1823 & 47.201 \\
$1766-67$ & 8.730 & & \\
\hline Totale & & & 9.892 .288 \\
\hline
\end{tabular}

cereali e legumi concesse, tanto per "infra" quanto per «extra Regno». Le serie hanno inizio dagli anni quaranta del XVI secolo e coprono un arco cronologico di tre secoli, sino agli anni venti del XIX secolo; per un totale di 651 volumi. I registri riguardanti il periodo $1720-1823$ sono tra quelli che presentano una maggiore omogeneità. Grazie a un totale di 272 volumi, disponiamo di una mappatura quasi completa delle licenze di esportazione per un secolo. 
gono» 125.096 salme. Il passaggio dalla dominazione austriaca a quella borbonica porta a un'intensificazione del commercio e i livelli di esportazione salgono sino alle 188.846 salme del 1737-38. Ma è con l'inizio degli anni '50 che i valori delle esportazioni tornano a livelli cinquecenteschi : nel 1751-52 partono dalla Sicilia 302.984 salme di grano; dieci anni dopo, nel 1764-65, le esportazioni arrivano a 365.755 e l'anno dopo a 440.267. La crisi dei raccolti del 1766 si ripercuote sulle esportazioni che scendono a sole 8.730 salme; ma il raccolto del '68 ne permette già la partenza di 184. 217. Tre anni dopo la quantità di grano per l'estero si porta a 271.178 salme per scendere a 43.701 nel 1771-72 e risalire l'anno successivo a 122.640. Gli avvenimenti del settembre 1773 - la rivolta palermitana contro il vicerè Fogliani - fanno scendere le esportazioni a 69.089 e successivamente a 16.089 salme. Dal 1776 al 1780 i valori tornano ad essere superiori alle 150 mila salme e toccano le 300 mila nel'81-82.

Paradossalmente è negli anni in cui il viceré Domenico Caracciolo concede la libertà dell'esportazione che i suoi livelli toccano il punto basso scendendo a 70.000 salme tra il 1782 e il 1785 . Alla vigilia della rivoluzione francese le esportazioni tornano a sfiorare le 300 mila salme di grano. Nel triennio successivo le quantità di grano per il mercato estero scendono sino a toccare il punto più basso del secolo con le 3.100 dovute alla carestia causata dall'invasione delle cavalette del 1792-93, per balzare l'anno dopo a uno dei suoi livelli più alti : 405.769 salme. Da questo picco via via diminuiscono le quantità di grano per «extra Regno", il 1796-97 è l'ultimo anno in cui si arriva a 162.435 salme; in seguito non si hanno valori superiori alle 100.000 salme. Anche se per il periodo non disponiamo della serie completa dei dati, quelli a disposizione mostrano un esportazione in media di 40.000 salme che salgono non oltre le 71.392 del 1818-19.

I caricatori siciliani possono essere divisi in tre gruppi : il primo riesce a immettere sul mercato oltre un milione di salme di frumento ognuno e ne fanno parte Girgenti, Licata, Termini e Sciacca; il secondo commercializza tra le centomila e il milione di salme e comprende Catania, Terranova, Pozzallo, Tusa, Marsala, Mazara e Trapani; con meno di centomila salme, del terzo gruppo fanno parte Palermo, Siculiana, Augusta, Roccella,
Vindicari, Agnone, Cefalù, Acireale, Taormina, Aci S. Antonio e Caronia (tav. 2).

Tav. 2 - Esportazione di grano dai caricatori dal 1720 al 1823 (valori in salme)

\begin{tabular}{|l|r|r|r|}
\hline \multicolumn{1}{|c|}{ Caricatore } & Infra regno & Extra regno & \multicolumn{1}{c|}{ Totale } \\
\hline Girgenti & 1.164 .009 & 3.667 .503 & 4.831 .512 \\
Licata & 770.978 & 1.569 .637 & 2.340 .615 \\
Termini & 238.406 & 1.464 .617 & 1.703 .023 \\
Sciacca & 458.221 & 1.223 .198 & 1.681 .419 \\
Castellammare & 389.716 & 642.159 & 1.031 .875 \\
Catania & 316.486 & 250.665 & 567.151 \\
Terranova & 182.840 & 192.163 & 375.003 \\
Pozzallo & 165.270 & 178.220 & 343.490 \\
Tusa & 182.404 & 132.515 & 314.919 \\
Marsala & 126.407 & 119.352 & 245.759 \\
Mazara & 108.781 & 67.353 & 176.134 \\
Trapani & 106.830 & 51.974 & 158.804 \\
Palermo & 7.870 & 91.883 & 99.753 \\
Siculiana & 50.545 & 23.871 & 74.416 \\
Augusta & 27.791 & 41.351 & 69.142 \\
Roccella & 26.272 & 28.033 & 54.305 \\
Vindicari & 18.998 & 22.107 & 41.105 \\
Agnone & 27.198 & 4.327 & 31.525 \\
Cefalù & 22.418 & 5.780 & 28.198 \\
Acireale & 4.052 & 11.770 & 15.822 \\
Taormina & 1.087 & 14.321 & 15.408 \\
Aci S. Antonio & 2.767 & 7.201 & 9.968 \\
Caronia & 316 & 2.697 & 3.013 \\
Altro* & 37.702 & 79.591 & 117.293 \\
\hline \multicolumn{1}{|c}{ Totale } & 4.437 .364 & 9.892 .288 & 14.329 .652 \\
\hline
\end{tabular}

* Caricatore non identificato

Dai magazzini e dalle fosse del primo gruppo di caricatori parte il $68 \%$ del grano diretto «infra Regno" e l'87\% di quello per "extra Regno». Rispetto al totale complessivo "estratto" dalla Sicilia nell'arco di un secolo, questi caricatori riforniscono $1^{\prime} 81 \%$ del mercato. Il $15 \%$ è invece rifornito dai caricatori del secondo gruppo che in un secolo commercializzano il $27 \%$ del grano per «infra Regno» e il 10\% per «extra Regno». Il terzo gruppo arriva appena a una quota del $4 \%$ del mercato complessivo che comprende il 3\% dei flussi di grano per l'interno e il 5\% di quelli per l'estero. Il grafico $n$. 1 dà bene l'idea delle diverse dimensioni di questi gruppi.

Nel primo gruppo di caricatori è Girgenti che ha la leadership : dal suo porto (l'odierna Porto Empedocle) lungo tutto un secolo partono 3.459.149 di salme di grano, che da sole coprono un terzo dell'intera esportazione della Sicilia. Inoltre con 1.164.009 salme di grano, Girgenti 


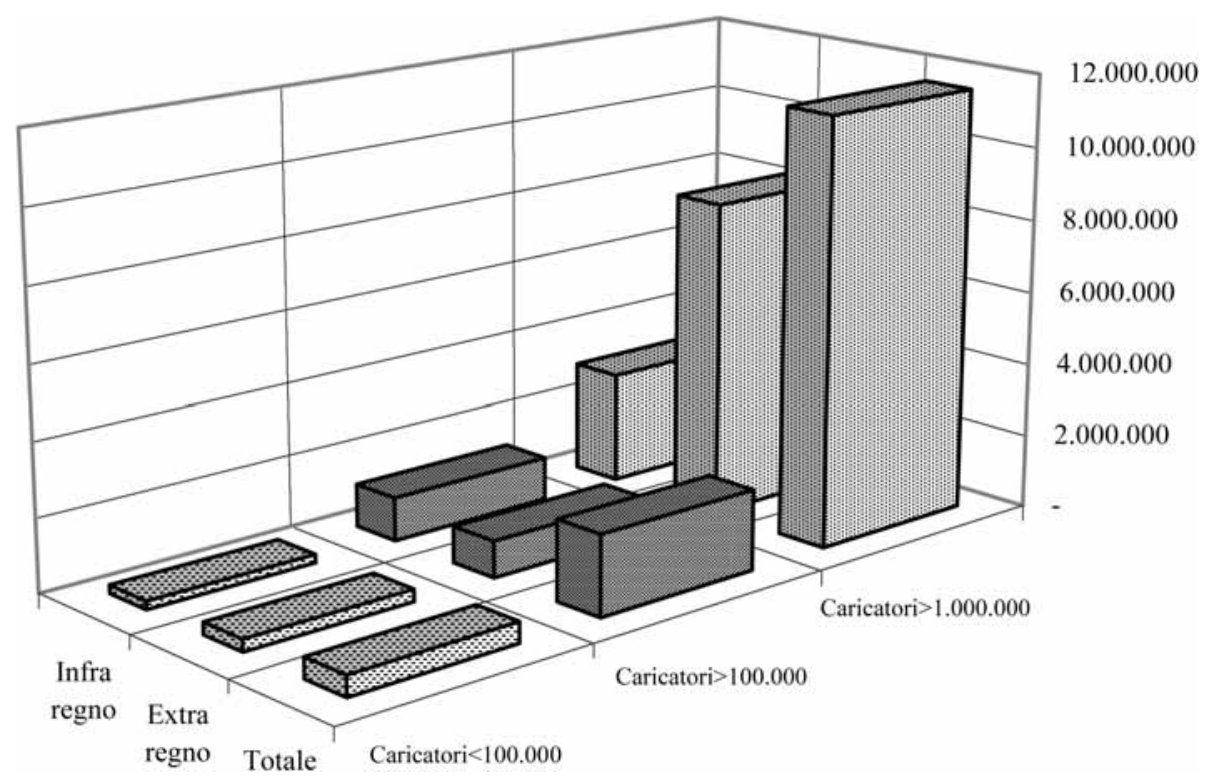

Graf. 1 - Esportazioni di grano divise per i tre gruppi di caricatori.

copre il $26 \%$ del mercato "infra Regno", e con 3.667 .503 il $37 \%$ di quello per "extra Regno». Nelle annate in cui si esporta meno, come il 1720-21, Girgenti fornisce al mercato estero più del 65\% del grano siciliano; e ancora nel 1761-64, quando dal suo caricatore parte per l'estero il $60 \%$ del grano siciliano. Nel 1766-67, quando le espor- tazioni toccano il punto più basso (25.157 salme), il $90 \%$ del grano riguarda Girgenti. Nel primo ventennio dell'Ottocento, a fronte di un inesorabile declino delle esportazioni, è ancora una volta dal suo caricatore che si preleva più del $70 \%$ del grano siciliano disponibile. Quando le annate sono buone si esporta con eguale intensità da tutti i

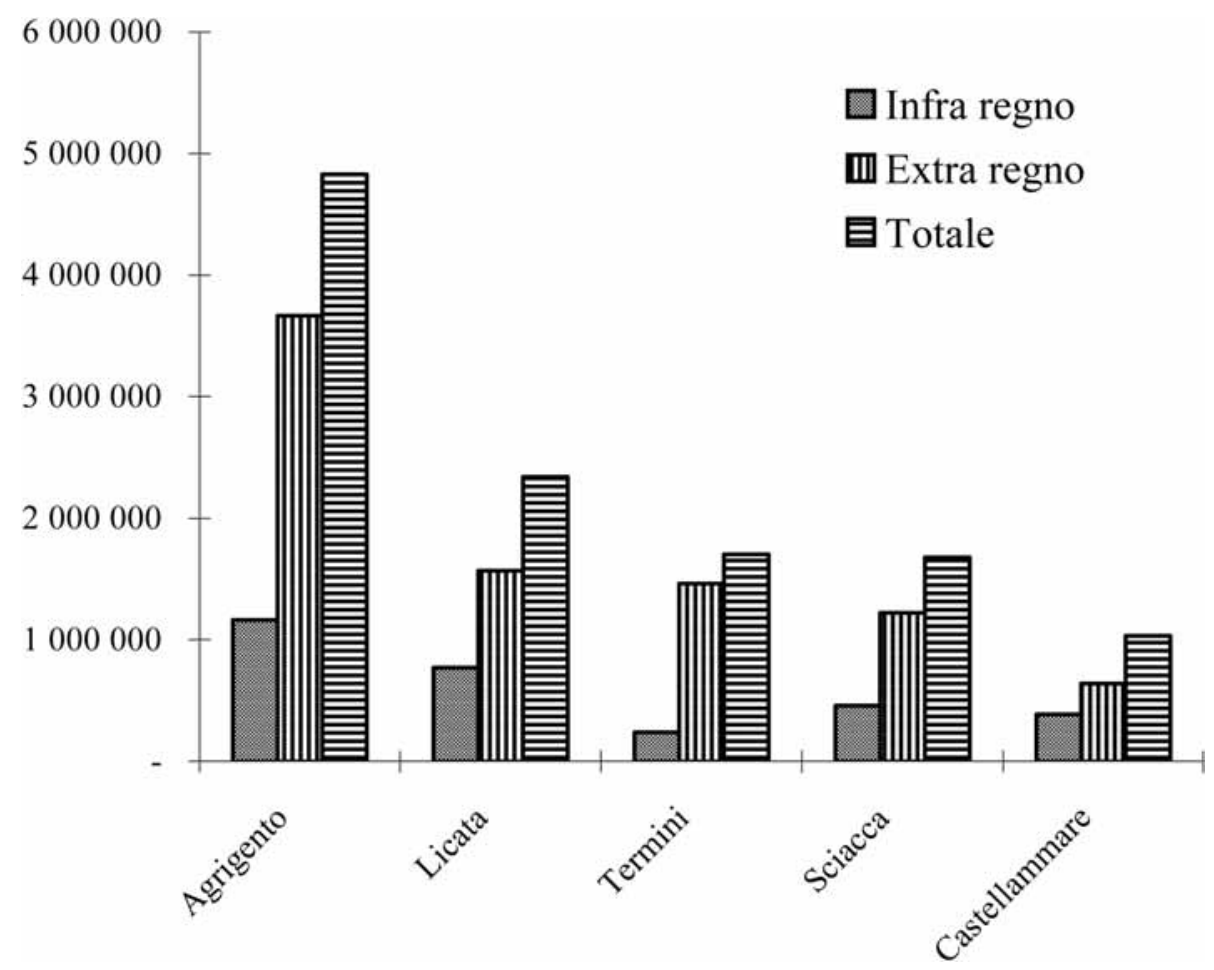

Graf. 2 - Caricatori che esportano più di 1.000 .000 di salme di grano (1720-1823). 
caricatori, ma è sempre Girgenti a confermarsi come il porto più importante per il mercato estero. Nel 1722-23 per l'estero partono 125.095 salme di grano e Girgenti ne fornisce il 50\%; l'anno successivo, su 128.828 salme esportate, dal porto di questa città se ne prelevano 41.819. Quando nel 1751-52 le esportazioni salgono sopra le 300.000 salme, il $31 \%$ è costituito dal grano agrigentino e sulle 440.267 salme del $1765-66$ ben 115.627 provengono da questa città.

Da Licata parte il $16 \%$ del grano per il mercato estero (1.569.637 salme), e il 17,3\% per quello interno (770.978). Nel 1735-36 su oltre 100.000 salme di grano per "extra Regno ", Licata da sola ne copre il $43 \%$ mentre Girgenti contribuisce con il $24 \%$ e Sciacca solo con l' $8,8 \%$. Pur se c'è qualche anno in cui Licata esporta quantità di grano superiori ad Girgenti, generalmente ne segue il trend generale, distanziata del 5-6\%. Quando il grano è poco ad Girgenti è poco pure a Licata e viceversa. Anche Sciacca partecipa allo stesso movimento, ma in posizione più arretrata, commercializzando il $10,3 \%$ del grano per «infra Regno» (458.221 salme) e il $12,4 \%$ di quello per "extra Regno" (1.223.198). In posizione diametralmente opposta ad Girgenti è Termini, al cui caricatore confluisce il grano delle Madonie e delle zone tirreniche che non hanno la produttività delle colline che si affacciano sul Canale di Sicilia. Da Termini partono 238.406 salme di grano per il mercato interno (pari al 5,4\%), e oltre 1.200 .000 per il mercato estero (pari al 12,4\% del mercato), poco meno di Sciacca. Dalla fine degli anni ' 40 il caricatore di Termini immette sul mercato lo stesso quantitativo di Sciacca o di Licata, superandoli diverse volte. Nella seconda metà degli anni '70 le esportazioni subiscono un crollo che dura sin all'inizio del decennio successivo quando si riportano di nuovo ai livelli precedenti. Castellammare è un caricatore che commercializza soprattutto nel mercato interno, del quale occupa una quota $\operatorname{dell}^{\prime} 8,8 \%$, pari a 389.716 salme; per l'estero partono quasi 650 mila salme che però occupano un segmento di mercato del $6,5 \%$. È nel trentennio tra gli anni ' 50 e ' 80 che più viva si fa la sua economia trainata, come vedremo, dalla domanda palermitana.

Se il primo gruppo di caricatori comprende solo città della Sicilia occidentale, nel secondo troviamo presenti porti disseminati lungo tutta le coste dell'isola.

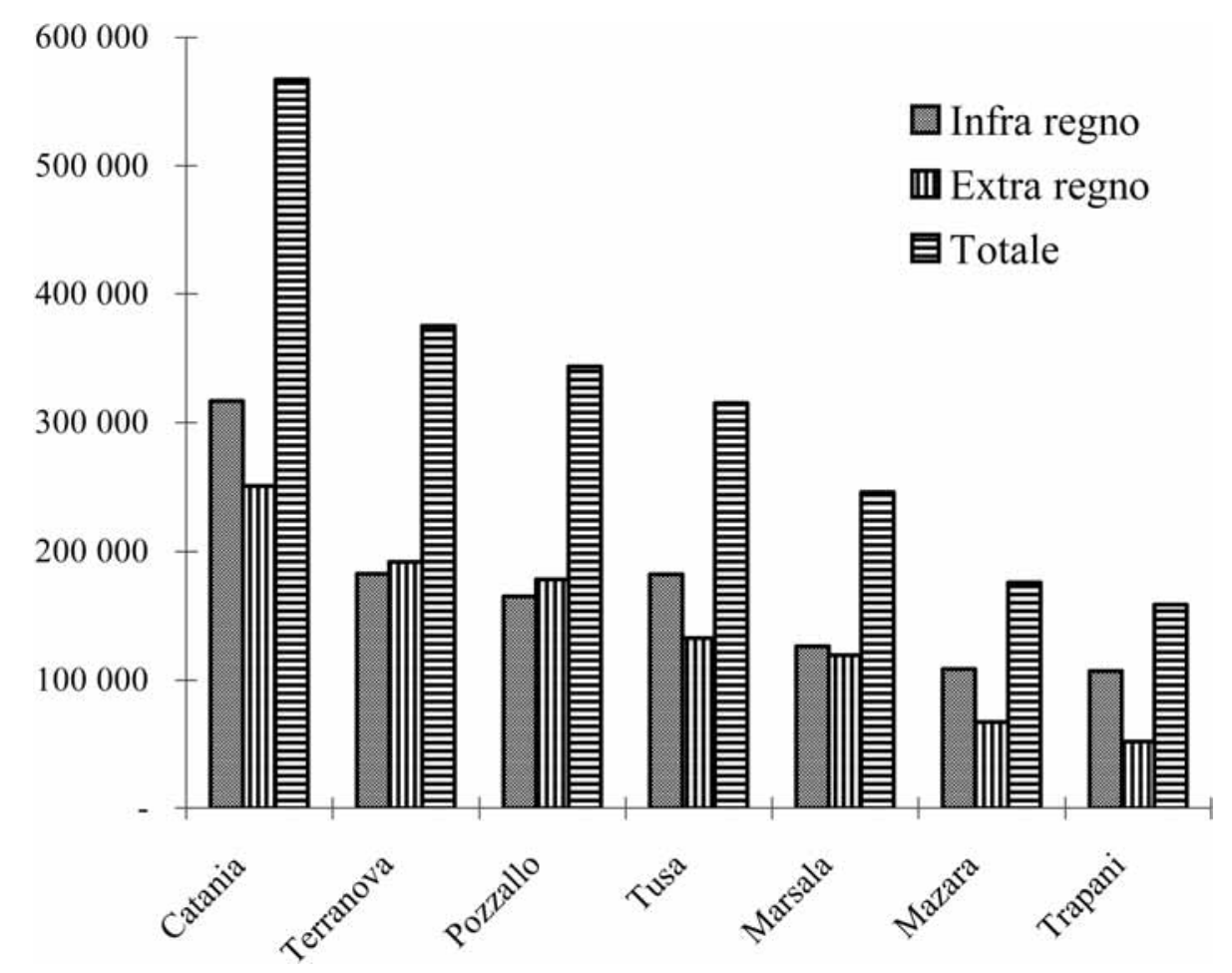

Graf. 3 - Caricatori che esportano più di centomila salme di grano (1720-1823). 
In questo gruppo Catania è il caricatore più importante. Commercializza in un secolo 316.486 salme di grano per «infra Regno» (il 7\% del mercato) e 250.665 per "extra Regno" (pari al $2,5 \%$ ). Sino alla metà del Settecento è uno dei porti più attivi del mercato interno, dove ha un accesso privilegiato Messina; tra gli anni '60 e '80 esporta molto anche sul mercato internazionale toccando un picco nel 1765-66, quando esporta più di 23 mila salme di grano.

Terranova, o Eraclea, occupa circa il $4 \%$ del mercato interno (182.840 salme di grano) e l'1,9\% di quello estero. È presente costantemente sul mercato e ha una improvvisa accelerazione dopo il 1800, quando riesce ad essere uno dei caricatori più attivi. In un clima di generale regresso, si nota per un segno contrario che porta Terranova a ritagliarsi fette sempre più grosse di mercato sino a occupare quasi il $30 \%$. Pozzallo è il caricatore della Contea di Modica, da dove partono il 3,7\% dei frumenti per «infra Regno» (165.270 salme) e $l^{\prime} 1,8 \%$ di quelli per l'estero (178.220). Nella prima metà degli anni '30 è tra i protagonisti del mercato interno con 15.896 estratte nel 1733-34. Inoltre l'anno dopo riesce a portare sul mercato estero quasi 17 mila salme di grano, il 9\% di tutti i caricatori. Tusa è il caricatore più importante dell'antica Val Demone, cioè della costa tirrenica del messinese, un territorio dominato dalla catena dei Nebrodi e dalla specializzazione nelle colture arboree ma povero di grano. Ma non tutto è dominio dell'albero: ed è significativo che Tusa riesca ad immettere sul mercato internazionale una quantità di grano (182.404 salme) pari a quella di caricatori presenti sulla costa opposta di Terranova e di Pozzallo, come pure di Marsala, Mazara e Trapani. Per «infra Regno» la sua quota di mercato arriva all'1,3\% (132.352 salme di grano) quasi interamente diretto verso le isole Eolie. Marsala, Mazara e Trapani hanno percentuali di esportazioni per «infra Regno» variabili al di sotto del 3\% e solo la prima riesce ad avere una quota del mercato internazionale superiore all' $1 \%$.

Nel terzo gruppo, che esporta meno di centomila salme di grano lungo l'arco di un secolo, vi sono i caricatori di Cefalù, Tusa, Caronia, Acireale, Aci S. Antonio, Agnone, Augusta e Vindicari, Palermo e Roccella. Le percentuali di esportazioni sia per infra sia per extra Regno non arrivano all' $1 \%$.

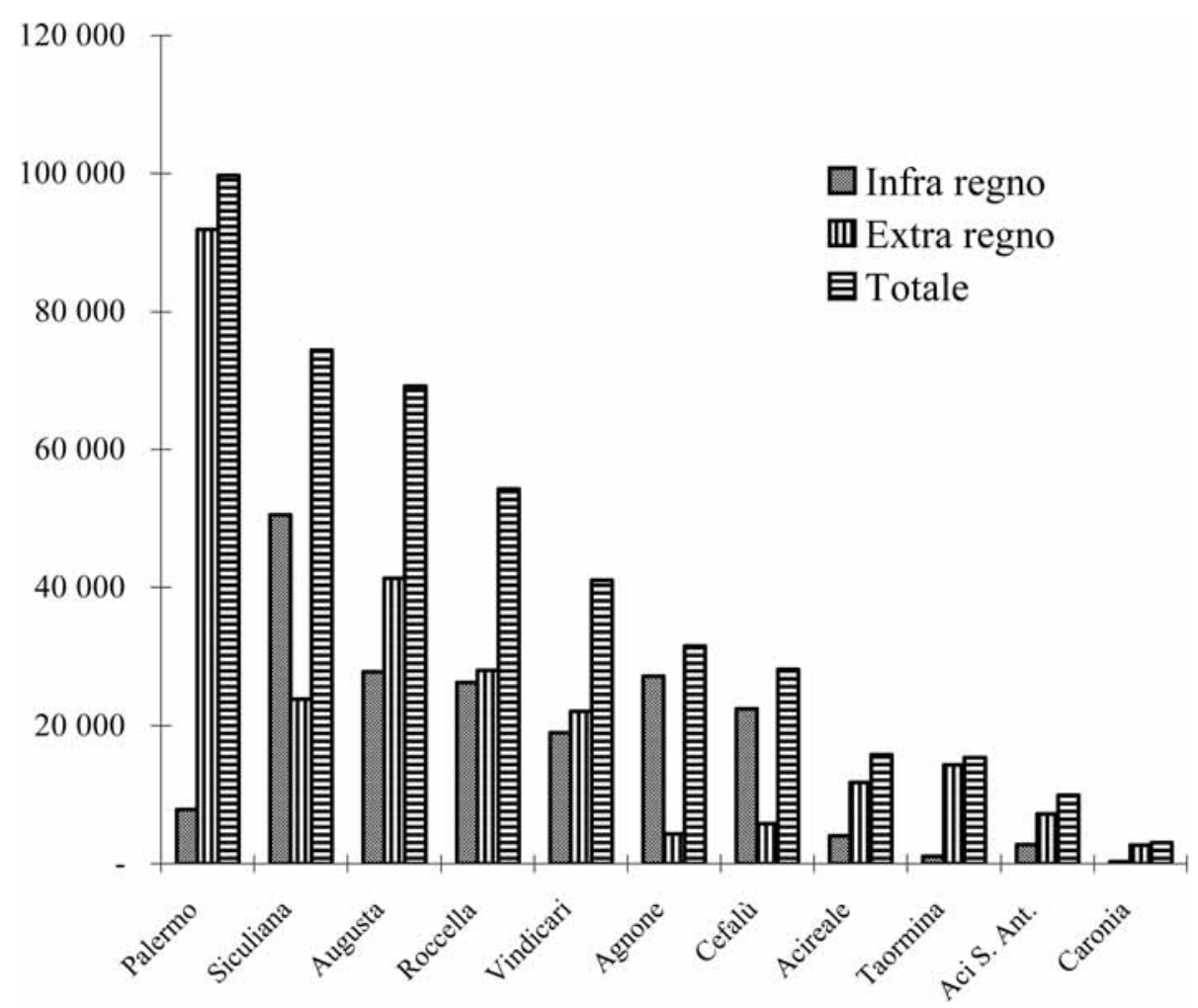

Graf. 4 - Caricatori che esportano meno di 100.000 salme di grano (1720-1823). 
Anche se i registri del Maestro Portulano non riportano la destinazione "extra Regno" - limitandosi a riferire che si tratta paesi "amici e confratelli» - dall'elenco delle nazionalità dei capitani si desume che i flussi di grano siciliano sono indirizzati verso i porti di Genova, Livorno, Marsiglia, Barcellona, Valencia. In anni di crisi il grano parte pure per Napoli, come nel 1759-60, quando è lo stesso Maestro Portulano Placido Notarbatolo duca di Villarosa a firmare, per ordine del re, permessi di estrazione per oltre 80.000 salme. A queste si sommano altre 2.500 salme concesse a Pietro Lignola «eletto del popolo di Napoli», 6.800 a favore di Gennaro Perelli per il rifornimento delle truppe napoletane e 1.200 a Baldassarre Ricci per il vettovagliamento della Marina. "Ma si tratta - secondo Paolo Macry - di congiuntura anomala e di movimenti mercantili egualmente eccezionali [...] Dalla Sicilia, il grano giunge alla città di Napoli solo in circostanze eccezionali» ${ }^{13}$. Ancora cause eccezionali sono quelle che fanno partire tra il 1793-94 oltre 400 mila salme di grano per Napoli, Amalfi, Livorno, Lisbona, Barcellona, Civitavecchia e Cadice. L'anno successivo 258.944 salme prendono le stesse direzioni, e sino al 1798 i caricatori della Sicilia continuano a rifornire questi porti. Ancora una volta il grano si rivelava un'arma strategica durante un periodo di conflitti. Tanto da far istallare, da lì a poco, i cannoni a difesa dei caricatori.

\section{E PER «INFRA REGNO»}

Nel Settecento l'elemento propulsivo dell'espansione del mercato del grano è la forte riprese demografica che investe tutta l'Europa. Un meccanismo simile a quello cinquecentesco. Anche in questo caso ci troviamo, come abbiamo visto, di fronte alla capacità della cerealicoltura siciliana di saper rispondere agli input che arrivano dall'esterno. Anche in Sicilia il XVIII secolo inaugura un ciclo accrescitivo che, con fasi alterne, si è protratto sino alla contemporaneità; così nel 1714 si conta una popolazione di 1.142.163, un secolo dopo si passa a 1.941 .566 (vedi graf. 5).

13. P. Macry, Mercato e società nel Regno di Napoli. Commercio del grano e politica economica nel Settecento, Napoli, 1974, p. 68.

14. I. Fazio, La politica del grano cit., p. 82.

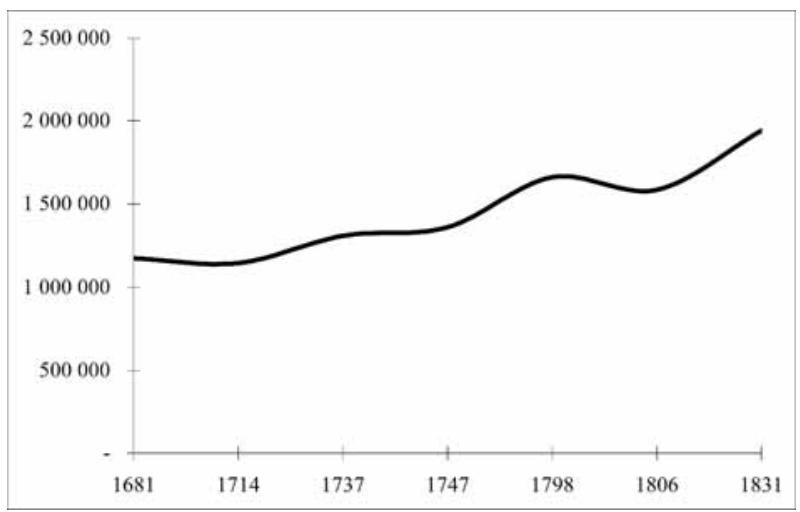

Fonte : nostra elaborazione su dati di G. Longhitano, D. Ligresti, S. Raffaele, M. Grillo e R. Nicotra, Studi di storia della popolazione della Sicilia del Settecento: una storia demografica europea, Catania, Società di Storia Patria per la Sicilia Orientale, 1979, p. 1.

Graf. 5 - Andamento demografico della Sicilia (1681-1831).

I registri del Maestro Portulano ci permettono di avere una mappatura completa delle esportazioni dai caricatori per «infra Regno», cioè per tutti gli altri porti e le isole minori della Sicilia. Inoltre dal 1751 viene considerata come «infra Regno" anche l'isola di Malta.

Tra il 1720 e il 1823 quasi quattro milioni e mezzo di salme di grano circolano via mare tra le coste della Sicilia : una media di 50.000 salme per anno (vedi tav. 3). Poca cosa rispetto ad una popolazione complessivamente in crescita, appena il $3-4 \%$ del fabbisogno generale. La maggior parte del grano arriva ai paesi dal loro circondario. La piccola comunità messinese di Santa Lucia studiata sotto la lente della microstoria da Ida Fazio - si rifornisce del 37,4\% di grano dai caricatori. Il ricorso a questo rifornimento è spesso privilegiato perchè "probabilmente il contatto con la domanda estera, almeno negli anni di tratta aperta, tende a moderarli [i prezzi], mentre negli anni di tratta chiusa, il frumento già affluito ai caricatori ha un prezzo più moderato $[. .$.$] perchè$ non può essere occultato per speculare sulla scarsità ${ }^{14}$. Gli anni di maggiore intensificazione del commercio per «infra Regno» corrispondono ad anni di tensione demografica come nel 1763-65, «la più lunga e complessa» crisi di mortalità siciliana del Settecento ${ }^{15}$.

15. R. Nicotra, Le crisi demografiche nella Sicilia del Settecento, in Quaderni del dipartimento di Scienze Storiche Antropologiche Geografiche dell'Università di Catania, n. 6, 1983, p. 42-43. 
Tav. 3 - Esportazione di grano per "infra Regno» (1720-1832) (Valori in salme $=222 \mathrm{Kg}$ )

\begin{tabular}{|c|c|c|c|}
\hline Anni & & Anni & \\
\hline $1719-20$ & 39.569 & $1771-72$ & 55.339 \\
\hline $1720-21$ & 79.721 & $1772-73$ & 70.131 \\
\hline $1721-22$ & 57.885 & $1773-74$ & 87.647 \\
\hline $1722-23$ & - & $1774-75$ & 119.267 \\
\hline $1723-24$ & - & $1775-76$ & 86.745 \\
\hline $1724-25$ & 81.002 & $1776-77$ & 62.605 \\
\hline $1725-26$ & 67.603 & $1777-78$ & 69.241 \\
\hline $1726-27$ & - & $1778-79$ & 85.767 \\
\hline $1727-28$ & 41.984 & $1779-80$ & 48.206 \\
\hline $1728-29$ & 79.708 & $1780-81$ & 49.632 \\
\hline $1729-30$ & 58.346 & $1781-82$ & 56.282 \\
\hline $1730-31$ & 40.565 & $1782-83$ & 52.014 \\
\hline $1731-32$ & 52.131 & $1783-84$ & - \\
\hline $1732-33$ & 60.963 & $1784-85$ & 66.697 \\
\hline $1733-34$ & 76.772 & $1785-86$ & 20.027 \\
\hline $1734-35$ & 54.136 & $1786-87$ & 42.765 \\
\hline $1735-36$ & 60.509 & $1787-88$ & 54.667 \\
\hline $1736-37$ & 58.594 & $1788-89$ & 33.511 \\
\hline $1737-38$ & 54.941 & $1789-90$ & 42.260 \\
\hline $1738-39$ & 54.027 & $1790-91$ & 56.142 \\
\hline $1739-40$ & 47.888 & 1791-92 & 47.086 \\
\hline $1740-41$ & 56.772 & $1792-93$ & 72.889 \\
\hline $1741-42$ & 55.506 & $1793-94$ & 41.865 \\
\hline $1742-43$ & 63.187 & $1794-95$ & - \\
\hline $1743-44$ & - & $1795-96$ & 36.878 \\
\hline $1744-45$ & 37.588 & $1796-97$ & 42.540 \\
\hline $1745-46$ & 31.636 & $1797-98$ & 43.610 \\
\hline $1746-47$ & 46.195 & $1798-99$ & 44.613 \\
\hline $1747-48$ & 50.149 & $1799-1800$ & 31.896 \\
\hline $1748-49$ & 35.794 & $1800-01$ & 50.188 \\
\hline $1749-50$ & 27.731 & $1801-02$ & 500 \\
\hline $1750-51$ & 39.432 & $1802-03$ & 17.184 \\
\hline $1751-52$ & 46.213 & $1803-04$ & 21.081 \\
\hline $1752-53$ & 75.214 & $1804-05$ & - \\
\hline $1753-54$ & 45.184 & $1805-06$ & - \\
\hline $1754-55$ & 90.595 & $1806-07$ & 22.692 \\
\hline $1755-56$ & 49.931 & $1807-08$ & 35.729 \\
\hline $1756-57$ & 48.761 & $1808-09$ & 37.941 \\
\hline $1757-58$ & 31.560 & $1809-10$ & - \\
\hline $1758-59$ & 42.659 & $1810-11$ & 28.083 \\
\hline $1759-60$ & 50.475 & $1811-12$ & - \\
\hline $1760-61$ & 64.892 & $1812-13$ & 34.113 \\
\hline $1761-62$ & - & $1813-14$ & 31.659 \\
\hline $1762-63$ & 50.489 & $1814-15$ & 41.151 \\
\hline $1763-64$ & 64.781 & $1815-16$ & - \\
\hline $1764-65$ & 43.946 & $1816-17$ & 9.883 \\
\hline $1765-66$ & 43.687 & $1817-18$ & 8.321 \\
\hline $1766-67$ & 82.180 & $1818-19$ & \\
\hline $1767-68$ & 73.513 & 1819 & 21.705 \\
\hline $1768-69$ & - & 1820 & 30.706 \\
\hline $1769-70$ & - & 1821 & 40.056 \\
\hline $1770-71$ & 48.889 & 1823 & 22.747 \\
\hline \multicolumn{3}{|r|}{ Totale } & 4.436 .864 \\
\hline
\end{tabular}

Nel 1760-61 le esportazioni per l'estero vengono chiuse a 11.194 salme mentre quelle per l'interno salgono a 64.892 e la stessa cosa avviene nei tre anni successivi, sin quando i buoni raccolti del '64 e del '65 rilanciano le esportazioni estere sino ai loro livelli massimi. Il circuito del mercato estero viene di nuovo isolato nel 1767-68, quando dai caricatori partono 73.513 salme di grano per i porti della Sicilia e sole 25.157 per «extra Regno». È negli anni settanta che le quantità di grano per «infra» raggiungono i loro tetto massimo; dal 1771 al 1774 si passa da 48.889 salme di grano a 87.647. Malgrado questa impennata della domanda interna le esportazioni per l'estero continuano ad avere un buon andamento, tanto da raggiungere nel 1772-73 le 122 mila salme. La rivolta di Palermo del settembre di quell'anno blocca le esportazioni del 1773-74 a 16.992 salme di grano mentre l'«infra Regno»schizza a 119.267, il picco più alto di tutto il secolo. Per gli anni successivi il livello del grano del circuito interno si mantiene al di sotto di quello estero. Solo nel 1792-93, ancora in presenza di una nuova crisi demografica, le tratte estere vengono chiuse e per l'interno si sale a 72.889 salme di grano in partenza dai caricatori. Il ventennio ottocentesco è all'insegna del grano per l'isola. Bisogna aspettare il biennio 1817-19 per vedere il circuito estero imporsi ancora su quello interno. È l'ultima fiammata di un grano che da questo momento in poi non solo non andrà più verso l'estero ma non basterà neanche più alla stessa Sicilia.

I luoghi di destinazione del grano per «infra Regno" sono soprattutto le isole Egadi, le Eolie, Pantelleria; alcune città del Val Demone come Milazzo e Patti; Siracusa sul versante ionico e Trapani su quello tirrenico. Queste due città ultime sono anche sede di caricatori che evidentemente nei momenti di crisi svolgono la funzione di "monti frumentari». Accanto a queste destinazioni, costanti per un secolo, vi sono altre città che richiedono grano, ma solo in momenti di crisi.

Esclusa Malta, dove arriva il 15\% del grano per «infra Regno» (pari a 661.667 salme), le percentuali degli altri porti di arrivo sono abbastanza basse. A Patti, Siracusa, Favignana giunge $1^{\prime} 1 \%$ del grano che circola all'interno della Sicilia. Il $2 \%$ sbarca a Trapani e il $6 \%$ a Milazzo. Nell'isola di Pantelleria, dove è presente una cospicua guarnigione militare, arriva il $4 \%$ del mercato. A Lipari, quasi interamente rifornita dal piccolo caricatore di Tusa, in un 
secolo arrivano 225.148 salme di grano, pari al 5\% dell'ammontare complessivo (vedi graf. 6).

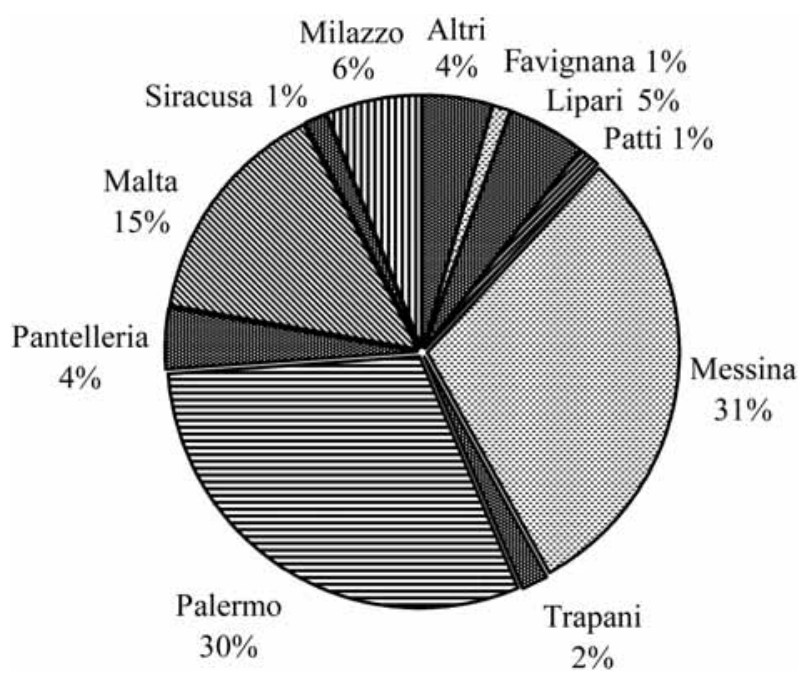

Graf. 6 - Destinazioni del grano per «infra Regno» (1720-1823).

In realtà i grandi mercati della Sicilia sono le sue due maggiori città : Palermo e Messina. Entrambe nel XVIII secolo hanno un forte incre- mento demografico, Palermo tra il 1714 e il 1831 passa da 115.000 abitanti a 173.478 e Messina da 60.848 a 83.772 (Vedi graf. 7). Quest'ultima è rasa al suolo da un terremoto il 5-7 febbraio 1783.

In un secolo sia a Messina sia a Palermo giunge quasi lo stesso quantitativo di grano, pari al $60 \%$ del mercato locale : poco più di 1.300 .000 salme. L'arrivo di grano segue il trend demografico delle due città. In una prima fase, dal 1720 al 1750, è Messina a ricevere più grano. Dai caricatori dipende il sostentamento, a volte, di oltre la metà della sua popolazione: nel 1728-29, 1737-38 e 1741-43 le arrivano più di 40 mila salme di grano per anno. Dopo incomincia una netta discesa del grano in arrivo, in coincidenza con il suo decremento demografico. Solo nel triennio 1773-75 le importazioni tornano sopra le 30.000 salme per abbassarsi sotto le 10.000 dopo gli anni '80 e le 5.000 dieci anni dopo. Il grano a Messina arriva soprattutto da Catania che in un secolo le invia quasi 300.000 salme di grano. Anche tutti gli altri caricatori della costa ionica (Aci S.Antonio, Acireale, Agnone, Siracusa, Taormina, Vindicari) inviano quasi la totalità del loro grano a Messina. Le arriva grano anche da Mazara, Marsala, Trapani

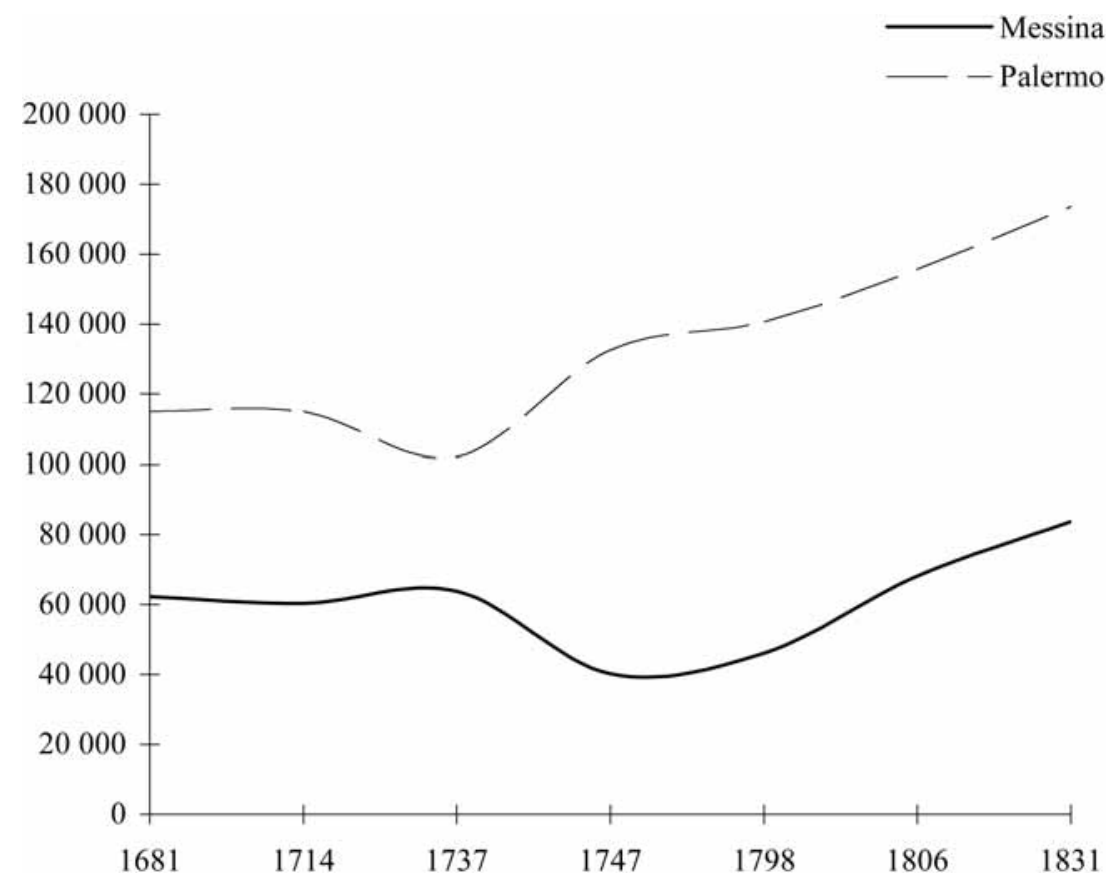

Fonte : nostra elaborazione su dati di G. Longhitano, D. Ligresti, S. Raffaele, M. Grillo e R. Nicotra, Studi di storia della popolazione della Sicilia del Settecento cit., p. 160 e 164.

Graf. 7 - Andamento demografico di Palermo e Messina (1681-1831). 
e Termini, ma sono ancora una volta Agrigento, Licata e Sciacca a fornire la maggior quantità di frumento (vedi tav. 4).

Tav. 4 - Caricatori che riforniscono Messina e Palermo (1720-1832) - valori in salme -

\begin{tabular}{|l|r|r|}
\hline \multicolumn{1}{|c|}{ Caricatore } & Messina & Palermo \\
\hline Aci S. Antonio & 2548 & 102 \\
Acireale & 2903 & 399 \\
Agnone & 22458 & 2542 \\
Agrigento & 268951 & 271222 \\
Augusta & 23472 & 1894 \\
Caronia & 80 & 198 \\
Castellammare & 24719 & 227511 \\
Catania & 299634 & 9910 \\
Cefalù & 1162 & 2258 \\
Licata & 271564 & 188108 \\
Marsala & 23137 & 61687 \\
Mazzara & 22577 & 66613 \\
Naso & - & 200 \\
Palermo & 3250 & - \\
Patti & - & 330 \\
Pozzallo & 61666 & 61164 \\
Roccella & - & 15830 \\
Sciacca & 164947 & 142615 \\
Siculiana & 900 & 11714 \\
Siracusa & 3595 & 40 \\
Taormina & 529 & 58 \\
Termini & 36380 & 50460 \\
Terranova & 33277 & 70108 \\
Trapani & 33859 & 19053 \\
Tusa & 6880 & 39117 \\
Vindicari & 13887 & 2814 \\
\hline & & 1346986 \\
\hline
\end{tabular}

A Palermo per il trentennio 1720-50 si assiste da un alternanza tra importazioni che al massimo toccano la punta delle 30.000 salme e al minimo scendono sino alle 5.000. Questa ritmicità di movimenti continua con un lento decremento sino al 1754-55, quando la città acquista oltre 42 mila salme di grano per venire incontro al fabbisogno di una popolazione in crescita. Da quest'anno in poi, si nota una ripresa costante che tocca le 46.204 salme nel 1766-67, restando in seguito sempre ancorata a valori, che dopo brusche discese, ritornano di nuovo verso le 30.000 salme. Quantità di grano che può soddisfare, al massimo, 1/4 dei suoi abitanti. Palermo, al contrario di Messina, gode di un retroterra agrario che le permette di rifornirsi di grano senza difficoltà. Inoltre ha un caricatore di cui viceportulano è il pretore della città stessa. E quindi formalmente sottratto al controllo diretto del Maestro Portulano. I suoi magazzini sono in grado di conservare 28 mila salme di grano. Anche in questo caso il caricatore svolge nei momenti di crisi la funzione di monte frumentario. Il grano a Palermo arriva soprattutto dai caricatori della costa occidentale : Castellammare del Golfo (227.222 salme), Agrigento (271.222), Licata (188.108) e Sciacca (142.615). Dalla costa tirrenica arriva pochissimo grano, poco più di 50 mila salme da Termini. Il resto partiva per Genova e Marsiglia, naturalmente prima della Rivoluzione.

Antonino Blando 


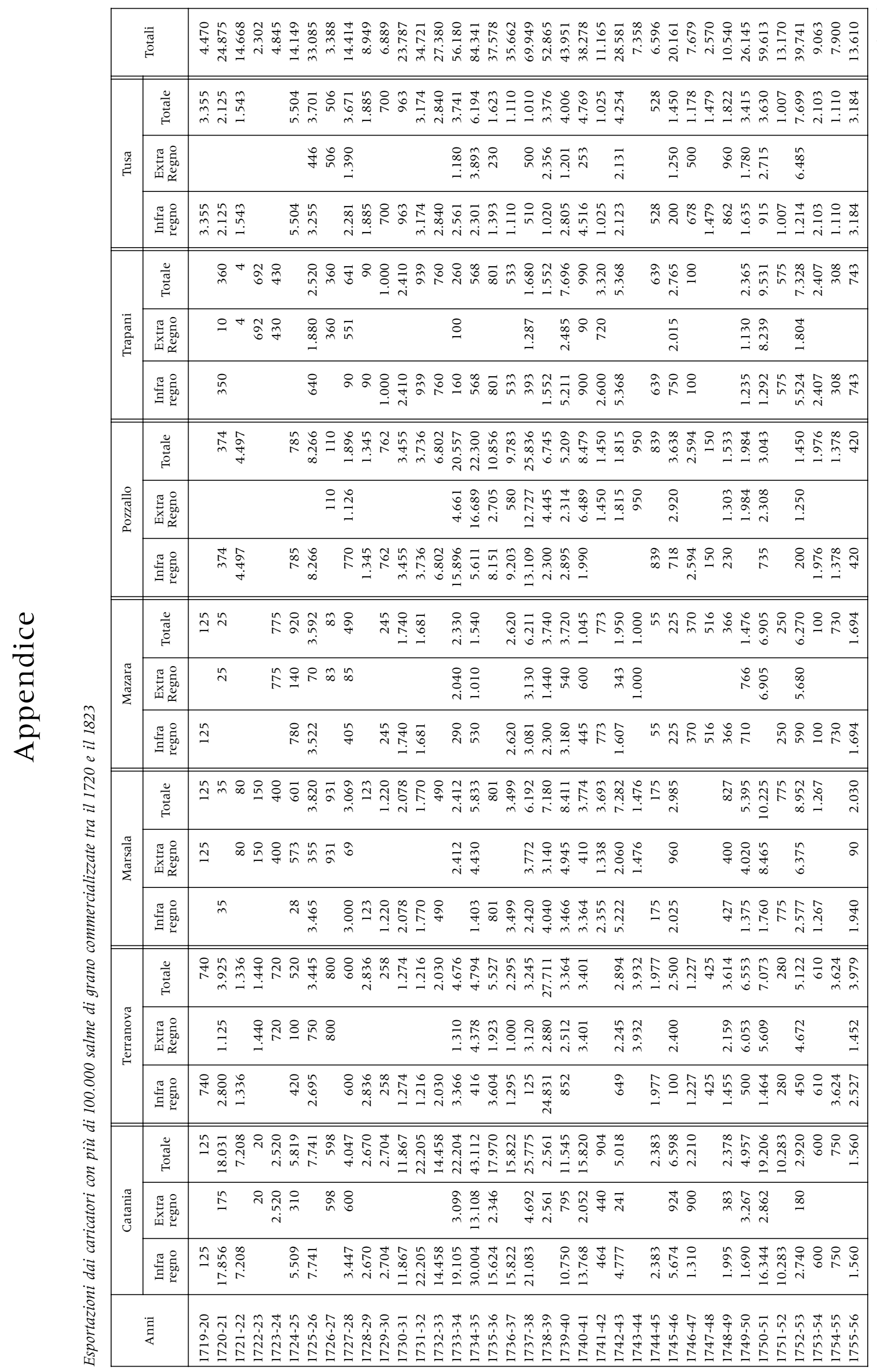




\begin{tabular}{|c|c|c|}
\hline \multicolumn{2}{|c|}{ 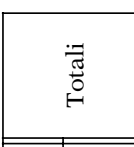 } & 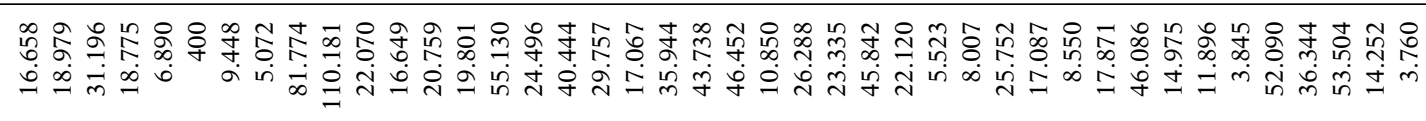 \\
\hline & $\frac{0}{\tilde{j}}$ & 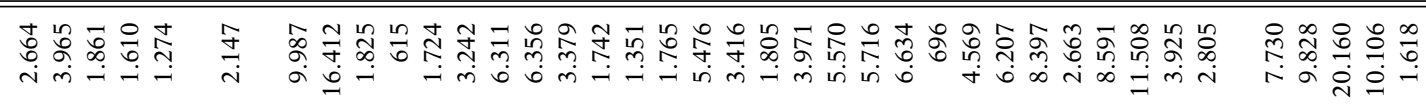 \\
\hline \multirow[t]{2}{*}{ thenta } & 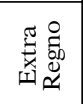 & 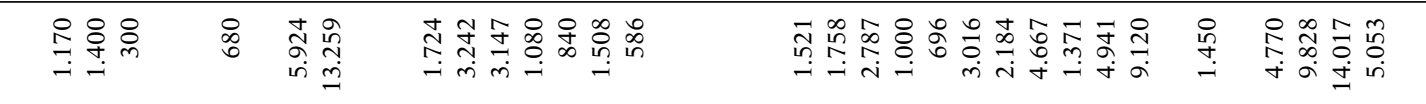 \\
\hline & 舀总 & 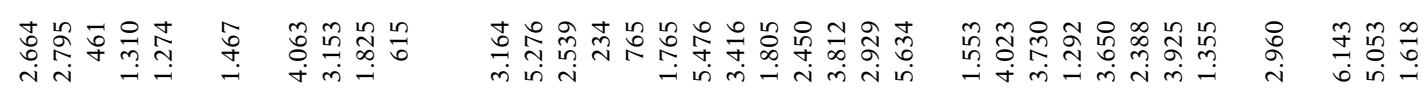 \\
\hline \multirow{3}{*}{ thenta } & 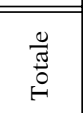 & 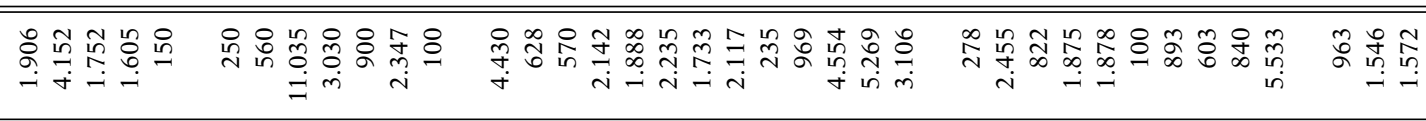 \\
\hline & $\begin{array}{l}\text { 舀总 } \\
\text { 品 }\end{array}$ & 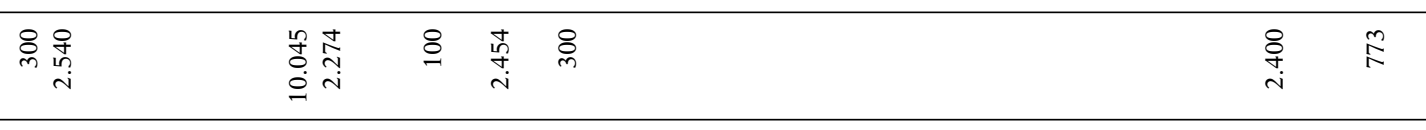 \\
\hline & 䶆总 & 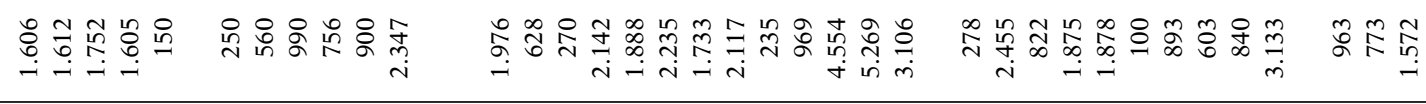 \\
\hline \multirow{3}{*}{ 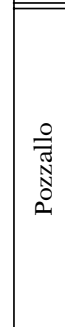 } & 惢 & 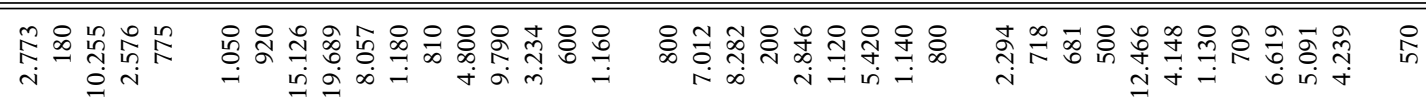 \\
\hline & 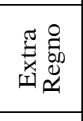 & 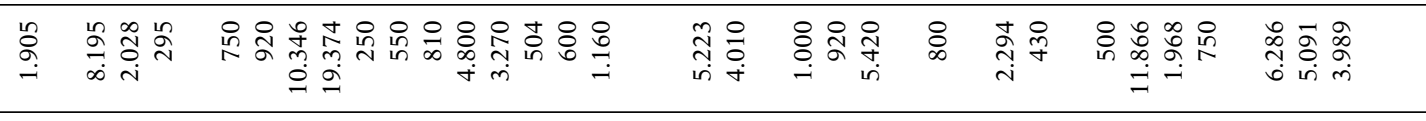 \\
\hline & 舀总 & 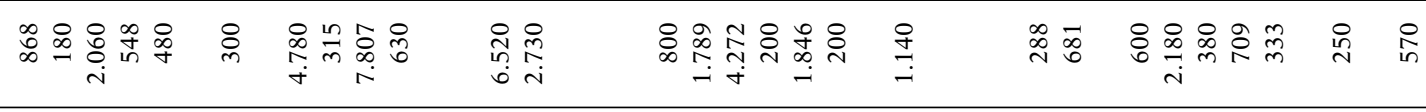 \\
\hline \multirow{3}{*}{ 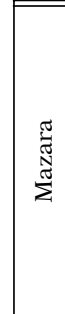 } & 尊 & 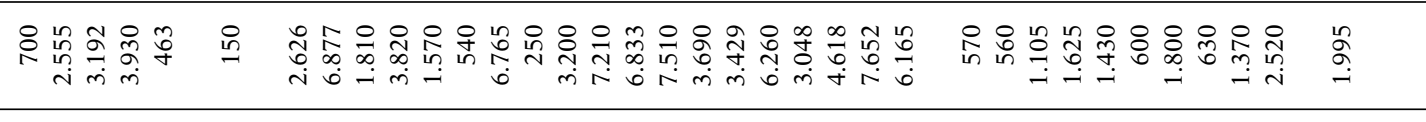 \\
\hline & 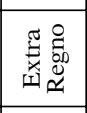 & 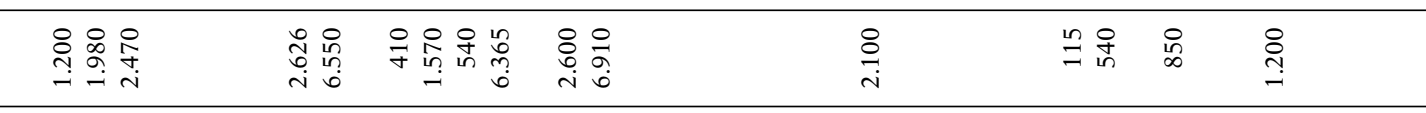 \\
\hline & 舀总 & 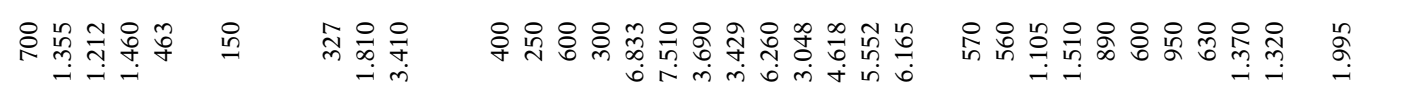 \\
\hline \multirow{3}{*}{ 要 } & 常 & 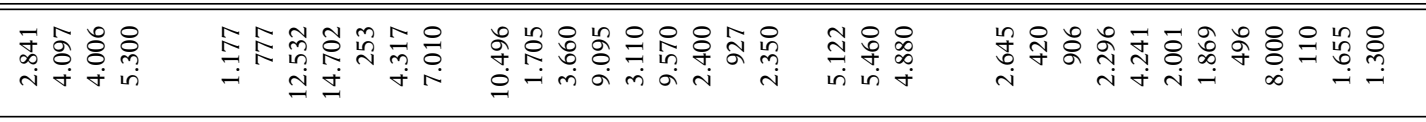 \\
\hline & 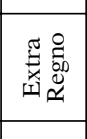 & 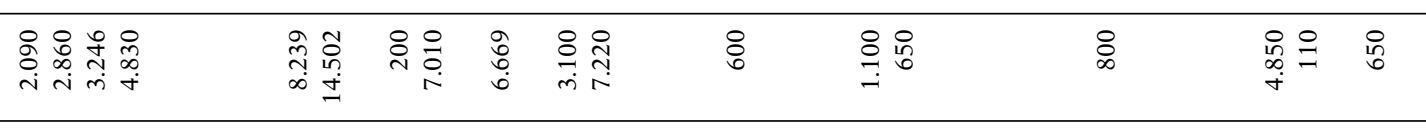 \\
\hline & 醀总 & 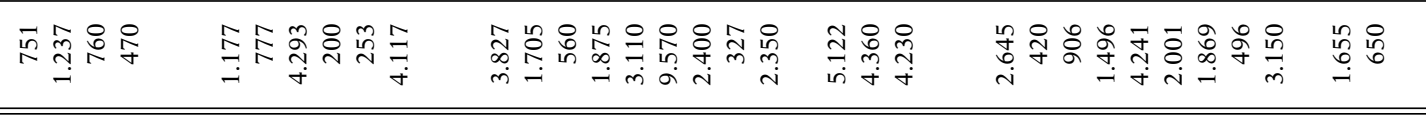 \\
\hline & $\frac{0}{\frac{0}{\omega}}$ & 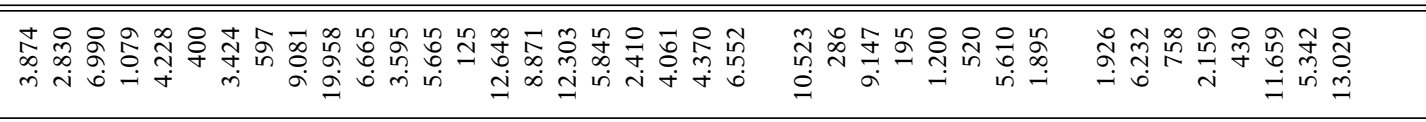 \\
\hline & 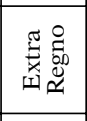 & 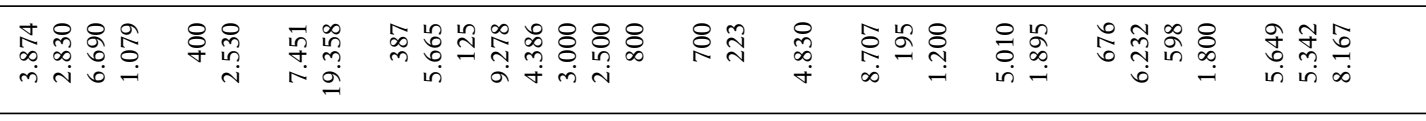 \\
\hline & 急总总 & \& \\
\hline \multirow{3}{*}{ 葋 } & 常 & 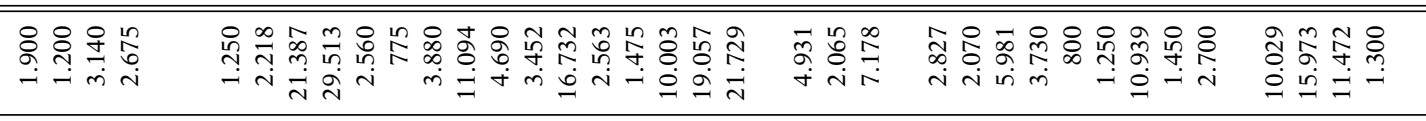 \\
\hline & 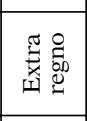 & 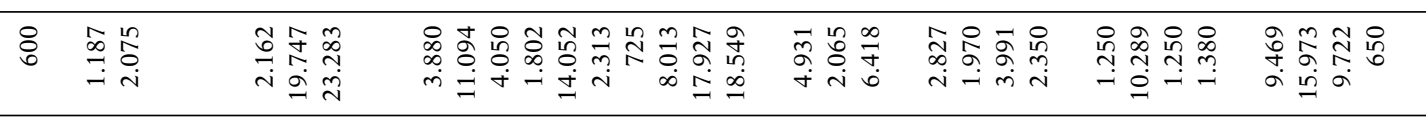 \\
\hline & 舀总 & 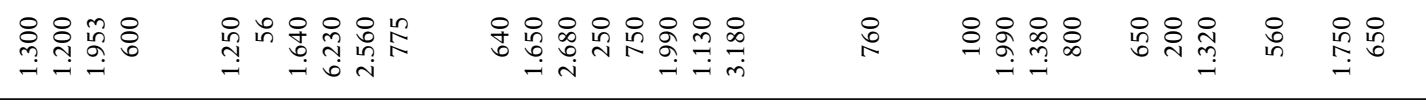 \\
\hline & E & 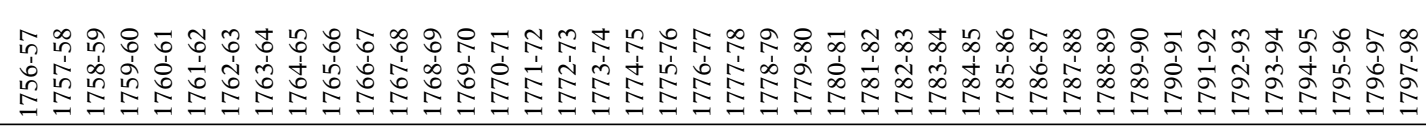 \\
\hline
\end{tabular}




\begin{tabular}{|c|c|c|c|}
\hline \multicolumn{2}{|c|}{ 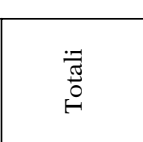 } & \multicolumn{2}{|l|}{ 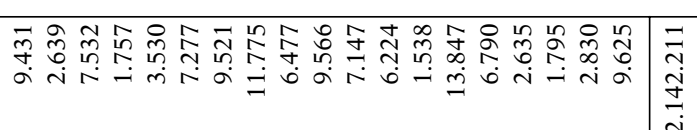 } \\
\hline \multirow{3}{*}{8} & 苞 & 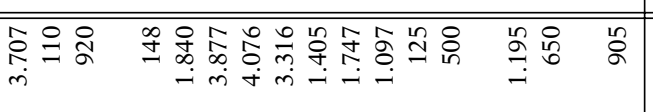 & 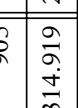 \\
\hline & 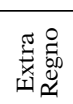 & in & \\
\hline & 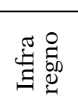 & 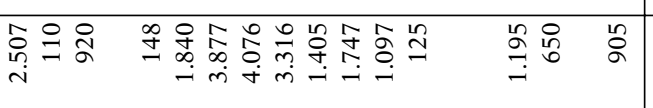 & 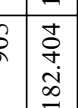 \\
\hline & 莺 & 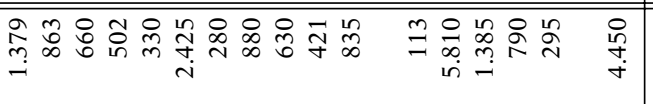 & $\begin{array}{ll}5 \\
\\
+\end{array}$ \\
\hline 镸 & 舀总 & 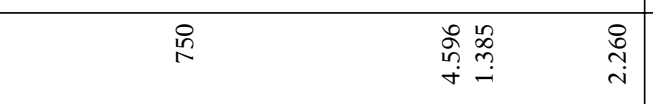 & is \\
\hline & 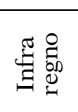 & 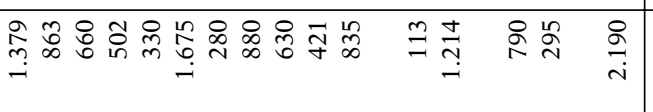 & 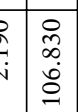 \\
\hline \multirow{3}{*}{ لِّ } & 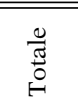 & 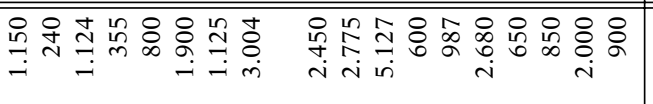 & 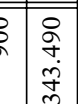 \\
\hline & 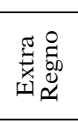 & in & \\
\hline & 舀总 & 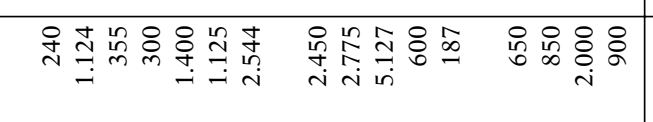 & 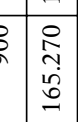 \\
\hline & 吾 & 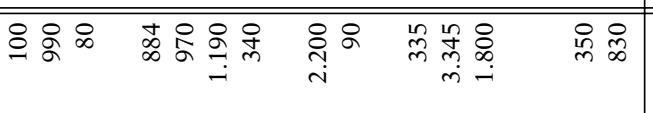 & i. \\
\hline 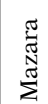 & 舀总 & 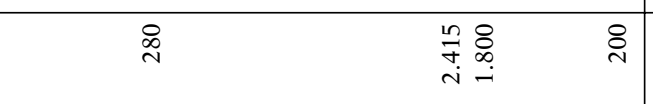 & \\
\hline & 舀器 & 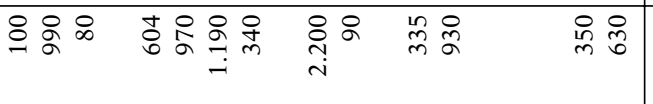 & 6 \\
\hline \multirow{3}{*}{$\stackrel{6}{=}$} & ב- & 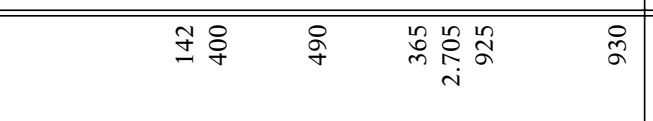 & \\
\hline & 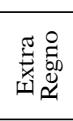 & 눙 & 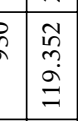 \\
\hline & 舀总 & İ \& & 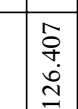 \\
\hline \multirow{3}{*}{ 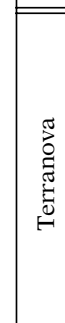 } & 竧 & 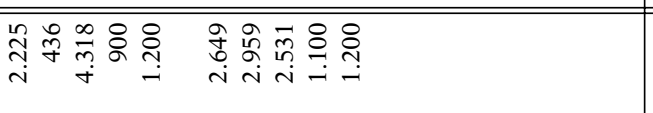 & \\
\hline & 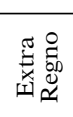 & ڤ్ & \\
\hline & 舀总 & 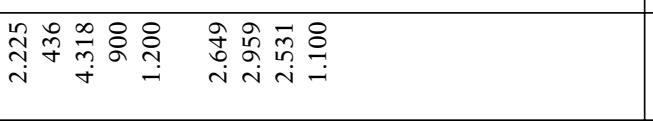 & 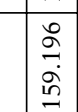 \\
\hline \multirow{3}{*}{ 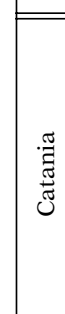 } & 楚 & 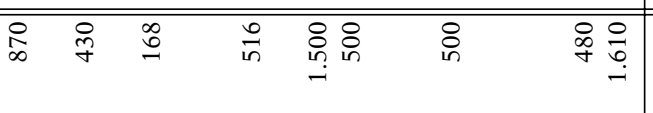 & : \\
\hline & 舀总总 & 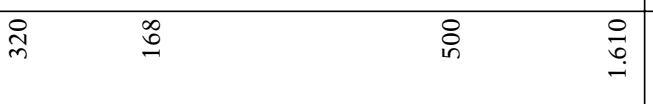 & 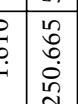 \\
\hline & 舀总 & in & $\int \frac{9}{2}$ \\
\hline & 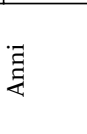 & 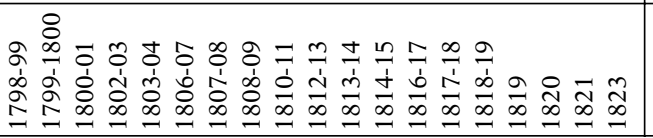 & \\
\hline
\end{tabular}




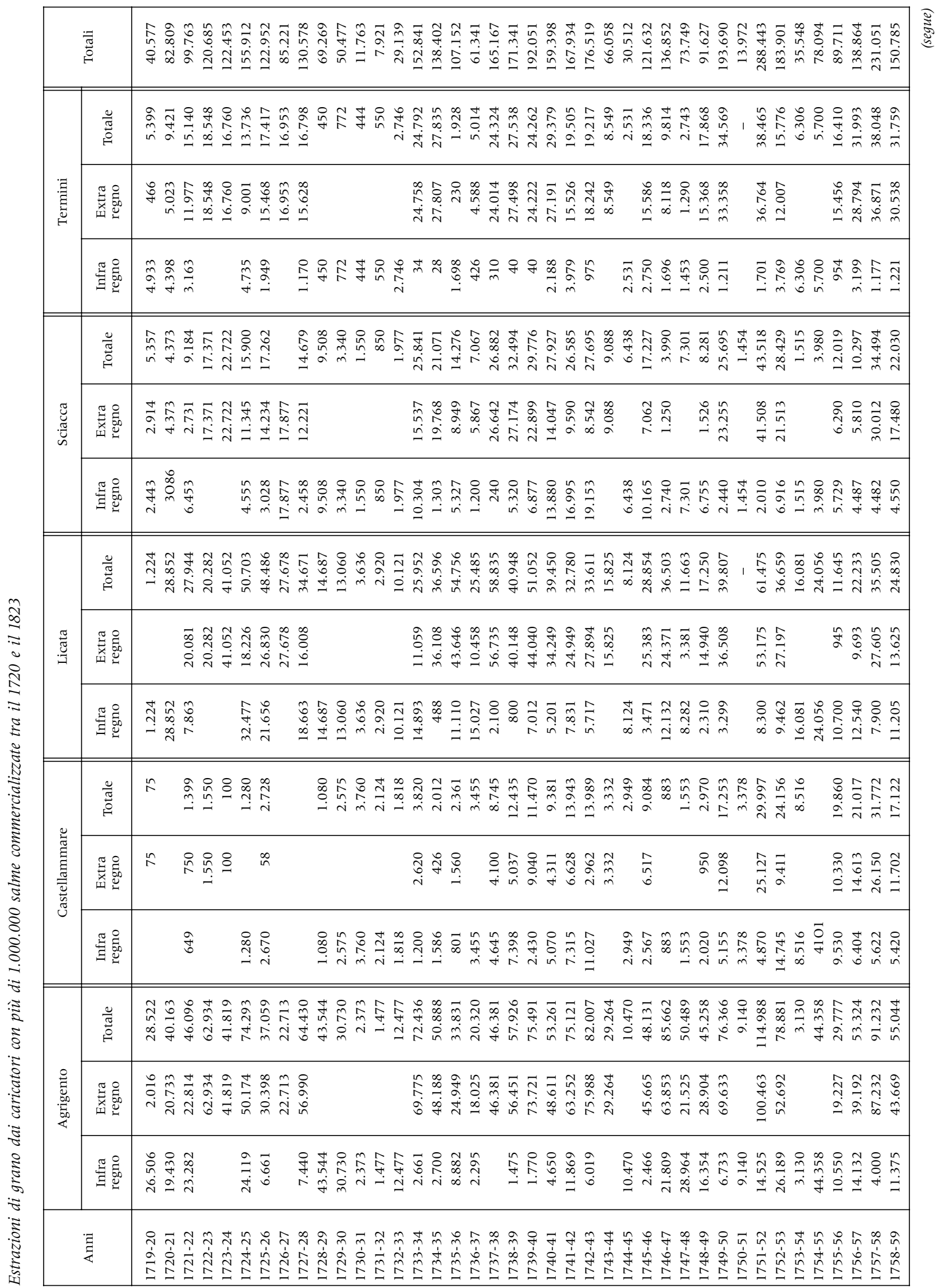




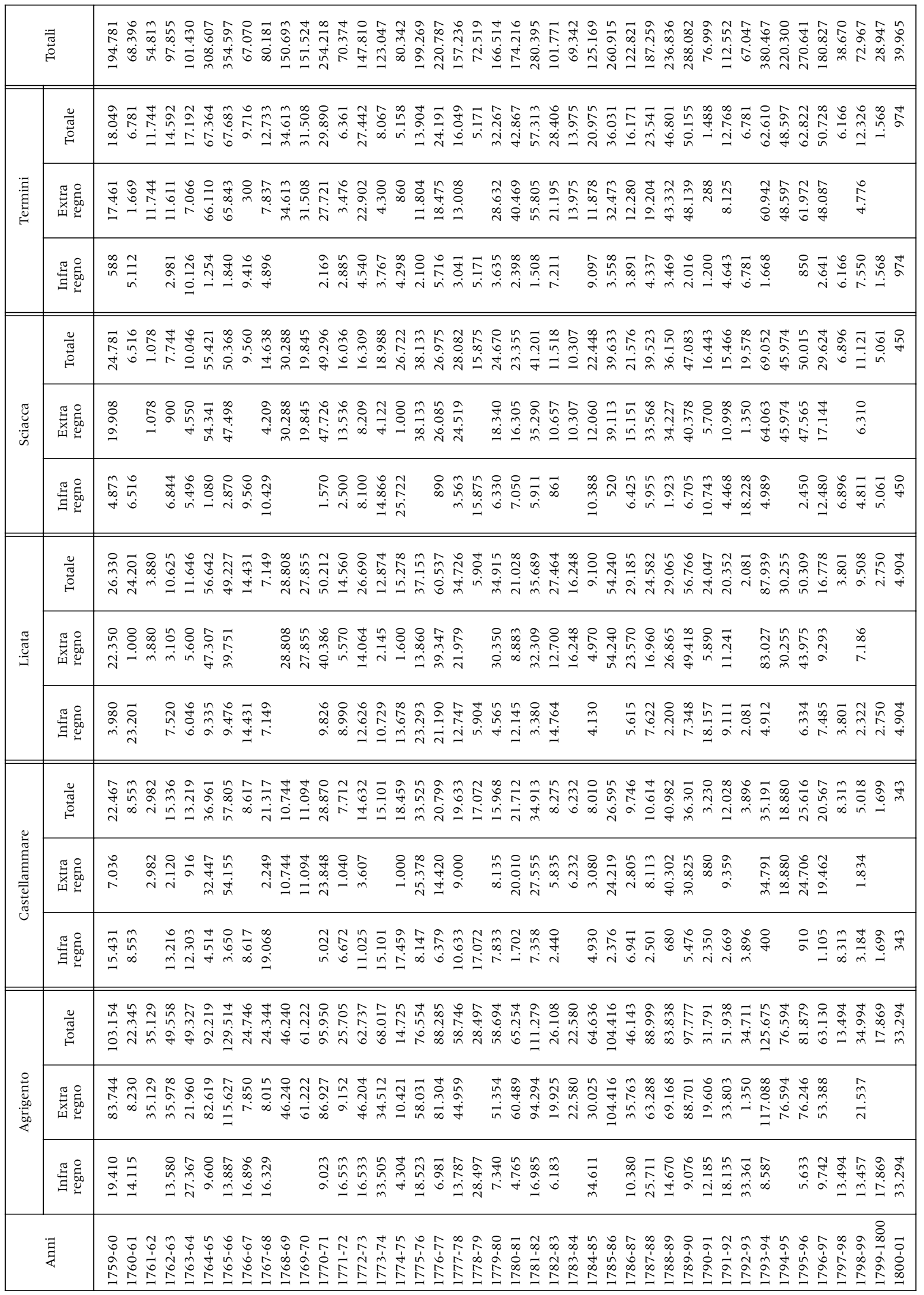




\begin{tabular}{|c|c|c|c|c|c|c|c|c|c|}
\hline & 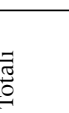 & 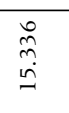 & $\stackrel{\tilde{n}}{i}$ & 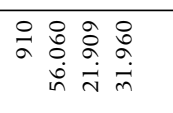 & 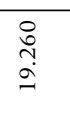 & 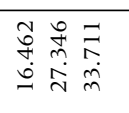 & 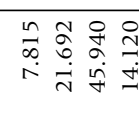 & 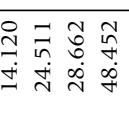 & 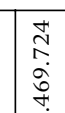 \\
\hline & 惢 & ì & 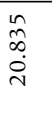 & 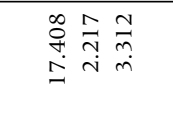 & $\underset{\vec{t}}{\vec{t}}$ & 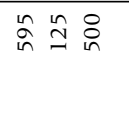 & 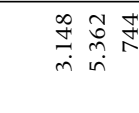 & $\$$ & 范 \\
\hline 咅 & 䍃㩊 & & 蒿 & 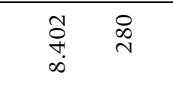 & & 品 & 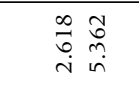 & $\stackrel{m}{\circ}$ & 惫 \\
\hline & 舀总 & ì & 恖 & 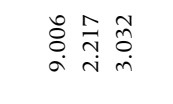 & $\vec{\sigma}$ & 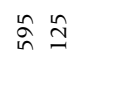 & in & $\stackrel{\circ}{\infty}$ & 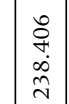 \\
\hline & 产 & & $\overline{\substack{\tilde{i} \\
i}}$ & 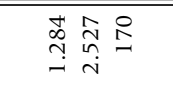 & $\stackrel{\hat{\tilde{\vartheta}}}{\underline{i}}$ & 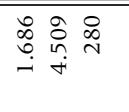 & 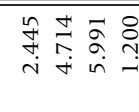 & 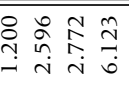 & 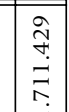 \\
\hline 营 & 妥总 & & & ir & & & 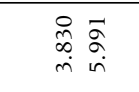 & ồ & 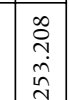 \\
\hline & 总总 & & î̀ & 专 & $\stackrel{\hat{a}}{-1}$ & 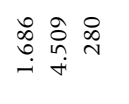 & 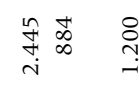 & 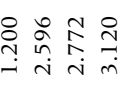 & $\left|\begin{array}{c}\vec{z} \\
\tilde{z} \\
\infty \\
\xi\end{array}\right|$ \\
\hline & 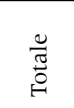 & $\begin{array}{l}\text { th } \\
\text { 总 } \\
0\end{array}$ & $\underset{7}{\Xi}$ & 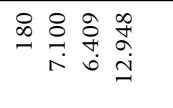 & 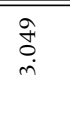 & 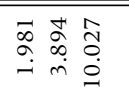 & 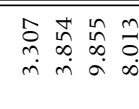 & 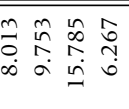 & 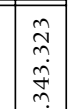 \\
\hline 㺃 & 妥总 & & î̀m & 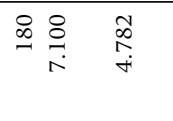 & & 高品 & 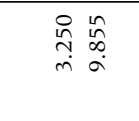 & 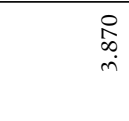 & 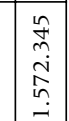 \\
\hline & 急总 & 苦 & 兑 & 亭 & 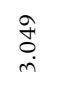 & 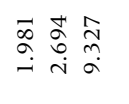 & 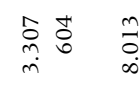 & 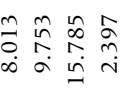 & $\left|\begin{array}{l}\infty \\
0 \\
0 \\
0 \\
1 \\
1\end{array}\right|$ \\
\hline & 愛 & & 尊 & 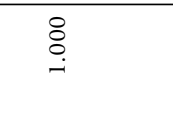 & & 苯等 & 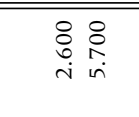 & 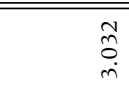 & 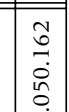 \\
\hline & 妥总 & & 尊 & 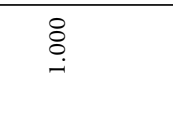 & & ঃ & 奋各 & \& & $\left|\begin{array}{l}0 \\
⿱ \\
0 \\
0 \\
0 \\
0\end{array}\right|$ \\
\hline & 畄善 & & \& & & & 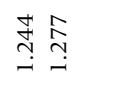 & & $\stackrel{\widetilde{N}}{\mathrm{i}}$ & 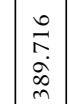 \\
\hline & 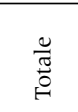 & 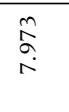 & 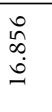 & 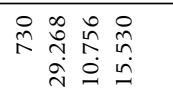 & 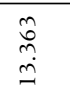 & 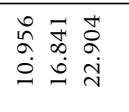 & 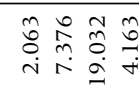 & 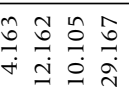 & 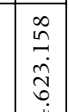 \\
\hline 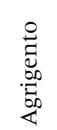 & 辤总 & & 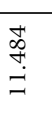 & 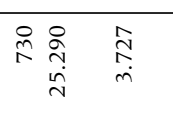 & & 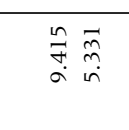 & 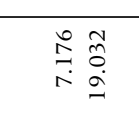 & 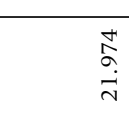 & 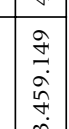 \\
\hline & 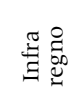 & 商 & م્ & 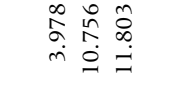 & $\begin{array}{c}\stackrel{0}{0} \\
\stackrel{0}{=}\end{array}$ & 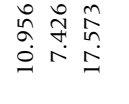 & क्ठ் & 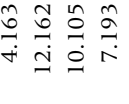 & $\left|\begin{array}{c}0 \\
0 \\
d \\
d \\
\hdashline \\
\hdashline\end{array}\right|$ \\
\hline & & & & & & & $\stackrel{\infty}{\infty}$ & $\underset{\infty}{\infty} \underset{\infty}{\infty}$ & 咅 \\
\hline
\end{tabular}

